\title{
Multi-participant Interaction in Multi-agent Naming Game
}

\author{
Wojciech Lorkiewicz, Radosław Katarzyniak \\ Wroclaw University of Technology, Institute of Informatics \\ E-mail: \{wojciech.lorkiewicz,radoslaw.katarzyniak\}@pwr.edu.pl
}

Received: 29 April 2013; revised: 3 June 2014; accepted: 5 June 2014; published online: 28 June 2014

\begin{abstract}
In this paper we analyse the influence of different interaction patterns on the behaviour of alignment processes in multi-agent Naming Game. We begin by introducing a meta-model of the Language Game that serves as a generalisation of the classical approach and facilitates better organisation and structuring of future research in the field. Further, we investigate the process against three interaction patterns (pair-wise, multi-speaker, and multi-hearer). The pair-wise interaction pattern involves a single speaker and a single hearer, participating in a single linguistic interaction; the multi-speaker and the multi-hearer interaction patterns assume multiple speaking and multiple hearing agents, respectively, involved in a single act of communication. All new types of interactions shape the performance of alignment processes that create the naming-convention. We show that the iteration-wise multi-participant patterns result in a visible improvement (increasing the number of speakers and the number of hearers results in a decrease of the number of interactions needed to reach a particular level of coherence), as compared to the classical pair-wise pattern. We show that an increase of the number of speakers and the number of hearers in multi-participant settings results in a decrease of the number of interactions, needed to reach a particular level of coherence.
\end{abstract}

Key words: agent systems, language game, semantic interaction

\section{INTRODUCTION}

Effective communication is a necessity in multi-agent systems. In particular, efficient ways of exchanging information are substantial and very important for embedded systems, such as autonomous agents. Moreover, these systems need to be flexible enough to handle the information exchange in unstable and evolving environments. Proper communication requires that all interacting agents use a well defined and shared set of individual names for objects about which the agents can and intend to "speak". As populations of embodied agents are usually located in at least partially undefined environments, it becomes impractical to assume that a complete and proper lexicon can be developed at the design time. Such approach would require an unrealistic assumption that all objects, both existing and possible to appear, should be foreseen by the designer in advance. Therefore it seems rational to develop a system comprised of autonomous agents capable of introducing new and aligning the already existing word-object pairings (associations between words and objects).
We model the ability to align new names as an internal learning mechanism, where the learning process occurs through a series of consecutive interactions between communicating agents. These interactions are designed in a way that leads the alignment processes to the formulation of a final coherent naming convention known to the entire population. We postulate that such a convention must be built up by agents from scratch. In particular, whenever agents encounter a new and unknown object, they should be able to associate it with a linguistic label. Thus, due to the inherent autonomy of agents, resulting individual naming conventions can differ in the population. Ultimately, the entire population must agree on a certain set of utilised word-object pairings. As such, through the learning process the wordobject associations should become more and more aligned, reaching a final status of a shared "global" naming.

We further assume that for an agent to acquire the proper interpretation of utilised language symbols, it needs to engage in multiple interactions with other agents. As in the embodied systems there are often no direct ways to transfer and 
investigate other agent's internal structures, the agent needs to learn word-object pairings only through interaction with the rest of population to which it belongs. We can note that an individual is faced with a tedious task. Each agent needs to develop a proper and coherent naming convention within the communicating group, solely by means of its private and local experiences. In consequence, the entire population needs to align the utilised language and formulate a consistent naming setup.

The general problem of language alignment in artificial systems is fundamental to the field of multi-agent systems. It is especially important during the design and utilisation stages of their embodied instantiations. For example, incorporating a flexible semantic communication system into a network of smart sensors may lower the system's energy utilisation and extend the system's operation time (see $[4,8]$ ). However, it should be stressed that the most significant and appealing seems to be the case of language alignment in robotic systems (see [16, 18]).

Developing a mechanism that would lead to a coherent formulation of names among interacting individuals is not a trivial task. Several approaches have been proposed and investigated in the literature to realize and/or study this task (see [3, 17, 20, 23]), ranging from associative types of memory (see [20]), through genetic algorithm models (see [23]), to neural network adaptations (see [17]). Among the aforementioned approaches the Language Game Model (the $L G M$ for short) (see $[19,21])$ seems to be the most popular and widely applied to practical utilisations of actual agents (including the embodied robots).

The LGM is a widely acknowledged model of language dynamics and seems to be a well grounded and the most promising framework in this field. It uses a simple routine type of interaction, associative name representation and cross-situational learning mechanism. It has been shown that in certain environmental settings the LGM leads to a coherent formulation of naming conventions within populations of interacting agents. In particular, the LGM has been focused on the development of intuitive and reasonable scheme of communication that allows a population of interacting agents to develop a shared lexicon (to reach a stable and coherent lexical convention). In this approach a population of agents engages in a series of pair-wise and conventionalised linguistic interactions. Moreover, an important aspect of this approach is its thrive to mimic a realistic interplay between individuals, where individual agents lack the direct ability to share internal representations (no-telepathy case), have only a restricted perspective of the system (no-omnipotence), and utilise solely the connection to their external surroundings (embeddedness).

For the sake of completeness, we note the research that falls outside of the original formulation of the LGM, namely: Baronchelli et al. (see [1]) and Lu et al. (see [15]), where different population structures (network topologies) were in- vestigated in a minimal naming game (single object environment); Lipowska et al. (see [13, 14]), where population turnovers and different population structures were investigated (an evolutionary approach to the vertical transmission of language); Loovern et al. (see [10]), where multiple word utterances were investigated; and the line of research related to different game types, for instance Action Game, Perspective Game, etc (with different types of objects).

The approach to be presented in the following sections is in line with the aforementioned LGM-based research and continues on our previous work, where we managed to define different attention-orienting strategies and studied their influence on the formation of coherent naming conventions (see [11]). In those works, we additionally explored the influence of different states of perception on the dynamic process of naming convention alignment (see [12]), whereas in this paper we investigate the process of name alignment and study the dynamic character of formation of coherent naming conventions in settings that fall outside of the original formulation of the LGM. Following the LGM-based approach, we extend this research by focusing on the influence of different interaction patterns on the performance of alignment processes in the multi-agent Naming Game (see [19]).

Using a simulated multi-agent system we aim to provide deeper insights into the effects of novel interaction patterns in the well known case of the least restrictive type of Naming Game, which is the naming game without feedback. Several questions should be addressed by our study, for instance: how our extensions affect the dynamics of the alignment processes and how the additional information about the environment is utilised by agents during the lexicon creation? Additionally, we investigate the performance of extended interaction patterns, namely: two originally proposed interaction patterns are evaluated against the classical simple pair-wise stance.

Our paper is organised into five major sections.

In the second section, we define the LG meta-model. In particular, we discuss the basic concepts and data structures used to conceptualize the most important elements of language games and propose their symbolic representation to support theoretical discussions as well as necessary technical implementation of simulation environments.

In the third section, we briefly discuss the model of language game, utilized by other authors (see Sec. II). In particular, we explain in what way this model is covered by our extended meta-model of language game. We pinpoint some limitations of previous research into the LG-based alignment, provide the reader with details of our own experimental settings, and explain in what sense they extend the basic pair-wise interaction pattern studied by other authors (see Sec. III).

In the fourth section, we define the simulation conditions utilised in our research, along with the baseline parameter values, and introduce necessary measures to describe a par- 
ticular state of the entire population of agents during the alignment.

In the fifth section, we present the simulation results (see Sec. V) divided into three groups: pair-wise (as the baseline for the analysis of extended interaction patterns), and two multiple participant patterns, one with multiple hearers (multi-hearer) and the other with multiple speakers (multispeaker), followed by a short summary (see Sec. VI).

\section{LANGUAGE GAME META MODEL}

\section{1. Structure and Pragmatics of LG-based Alignment in Multi-agent Systems}

In the LGM the game played between the agents is governed through a series of consecutive individual episodes. Each episode involves two randomly selected agents: one acting as a speaker, and the other as a hearer. Both are situated in the same state of an external environment (common context). In such setting the speaker tries to draw attention of the hearer to a particular element in a shared scene (intended topic), using a linguistic clue (utterance). Further, the hearer tries to correctly interpret the linguistic utterance as a particular element of the current state of the external environment. It should be underlined that the agents do not receive any form of direct feedback concerning the outcomes of the game. The interpreted meaning and the heard word pair (word-object pairing) is regarded as the most probable one. Moreover, there is no per se idea of success of the communication. The speaker provides solely a clue to the hearer and has no knowledge of the outcome of such interaction. Whereas the hearer has only very limited information (i.e. a glimpse on the speaking agent's stance that refers to a single word and a single object that is additionally noisy, depending on the context size). As such, after a single interaction the hearer is still unable to assess the quality of a particular word-meaning mapping.

In essence, the symbols utilized by the agents can be correlated with a particular internal reflection of an empirically perceived and external state of the environment (population's source of the grounded meaning) (see [7]). Through numerous interactions the learning agent is capable of identifying the correct word-object pairing as sources of meaning for each encountered symbol of the language. The essence of the idea incorporated in the LGM is hidden in applying the hearer's internal linguistic processes over multiple learning episodes. Consequently, after receiving enough samples the agent is able to build adequate correlations between utilised linguistic symbols and observed states of the external environment.

The starting point for our research is defined by a naming game without feedback ${ }^{1}$, where it is assumed that neither the hearer nor the speaker is able to provide a non-verbal clues one to each other. Consequently, it should be stressed that in such a game neither of the agents has a direct information and means to determine whether the interaction was successful or not. The success of communication can only be elaborated on the system's level of analysis.

The incorporated (agent-based) model of the system is a complex one. It consists of several sub-models that define particular aspects governed within the process of resolution of language meaning (similar to $[11,12]$ ). In order to define a particular case of the LG-based language alignment we need to consider a particular set of detailed conceptual components, corresponding to the following, detailed submodels: environment, population, agent, reflection of current environment, constraints to perceptions, and interaction rules.

The meta-model to be presented in this section captures a generalised view on the language game approach to the alignment of naming conventions between agents in a given population. In particular, the main goal of this meta model is to define a general structure and main components of the task, as well as provide the approach with a formal definition of a multi-agent system capable of engaging in language games.

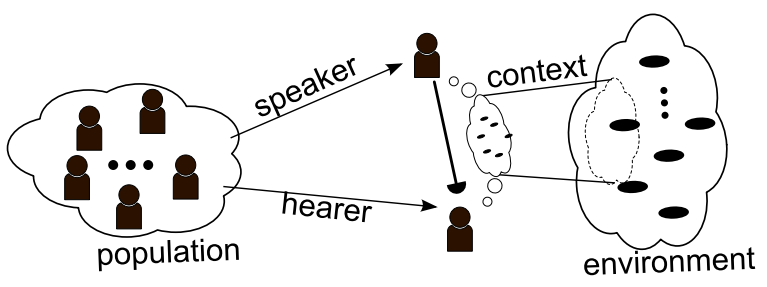

Fig. 1. Language Game

\section{2. Environment \& Population}

In our approach we follow the cognitive stance that the environment-individual interplay constitutes the ultimate source of meaning and results in particular "mental" conceptualisations in individual agents. The environment is treated as a common ground of interaction, shared between the agents and built from invariants, where each invariant is represented by a unique identifier (' $\left.i d_{i}{ }^{\prime}\right)$. At particular moments each invariant may be exposed to all or some of autonomous subjects as an objectively existing and distinguishable part of the world. All invariants possible to be created in LGM system constitute the environment as the whole.

\section{Definition 1 (Environment)}

The environment $\mathcal{E}$ is given as the space of all distinguishable invariants of the objective world. It is formally represented by the following set: ${ }^{2}$

\footnotetext{
${ }^{1}$ Such an approach assumes that there is no direct channel of communication between the agents that allows agents to exchange the feedback information.

${ }^{2}$ We further assume that function $\|\|:. X \rightarrow \aleph$ for a given set $X$ denotes the cardinality of the set $X$.
} 


$$
\mathcal{E}=\left\{i d_{1}, i d_{2}, \ldots, i d_{\|\mathcal{E}\|}\right\} .
$$

and constitutes the ultimate source of meaning available to agents in the LGM system.

The dynamic aspect of the environment $\mathcal{E}$ is captured by momentary availability of certain invariants in its current state. This availability is modelled through the notion of context (see Def. 6), given by a subset of $\mathcal{E}$ consisting of currently exhibited and accessible invariants. From a practical point of view, the context defines certain situations for currently interacting agents and serves as the only entity, though complex, objectively shared between participants of each linguistic interaction. Certainly, all internal representations of invariants, which are the figments of the agent's perception, can and do differ in the ontological sense. However, they all refer to the same "physical" entities that are external to particular agents in the LGM system (see [9]). The notion of context is modelled by the context function, given as follows:

\section{Definition 2 (Context)}

For all time points $t \in \mathcal{T}$ the context function

$$
\mathcal{X}^{\mathcal{E}}: \mathcal{T} \rightarrow 2^{\mathcal{E}}
$$

is defined. For a given time point $t \in \mathcal{T}$ this function yields the actual context $\mathcal{X}^{\mathcal{E}}(t) \in 2^{\mathcal{E}}$, in which the interacting agents are situated, given as a set of invariants currently available for agents' perception.

Two features of $\mathcal{E}$, formal and pragmatic, need to be stressed. First, it is important to note that identifiers of invariants are available only at the system's level, and are inaccessible internally for particular agents' minds. Second, the environment is static and finite in the sense that its distinguishable parts are constant and constrain the actual limits of its possible quantisation.

Regarding the agents available in the system, all are assumed to be embodied and situated in the environment (see [17]). They are assigned unique identifiers, too, and are called the population:

\section{Definition 3 (Population)}

The set of all agents available in the system is called the population $\mathcal{P}$. The population and its members are formally denoted by the following symbols:

$$
\mathcal{P}=\left\{a_{1}, a_{2}, \ldots, a_{\|\mathcal{P}\|}\right\} .
$$

The population is static and finite in the sense that all agents are known beforehand, and there are no particular ways to introduce new agents during the system's runtime.

It is assumed that all individual agents are capable of developing subjective and internal representations of invariants of the environment $\mathcal{E}$, by means of dedicated and embodied mechanisms of perception. As aforementioned, the environment is built from invariants that are represented by a set of unique identifiers (' $i d_{i}$ '). Each such invariant is external to the entire population, and each individual agent alone. Consequently it is directly unavailable to the agent's higher internal processing. The invariant is, however, directly accessible by the agent's strictly private perception mechanism. A particular invariant in the external environment triggers a particular reaction of the agent's perception that is further propagated to higher level cognitive processing. As such, individual perception mechanisms are able to create certain internal projections/reflections of the external world that are directly accessible to the agent. In essence a particular invariant in the external environment can trigger a set of internal reflections that are activated within the agent and are further available to its higher cognitive processes. This natural process is modelled by the notion of internal reflection mapping (see Def. 4):

\section{Definition 4 (Internal Reflection)}

Each agent $a \in \mathcal{P}$ is able to register an external invariant through a notion of internal reflection $\left.o_{i}^{a} \in \mathcal{E}\right|_{a}$ assigned by a relation $\mathcal{R}^{a}$ :

$$
\mathcal{R}^{a} \in \mathcal{E} \times\left.\mathcal{E}\right|_{a},
$$

where $\mathcal{R}^{a}$ is the embodied mechanism of invariants' perception, and $\left.\mathcal{E}\right|_{a}$ is the set of internal reflections.

Remark: It should be noted that for the sake of simplicity we further assume that $\mathcal{R}^{a}$ is a function, i.e. it may happen that any single invariant can be mapped to and only to a single internal reflection. However, $\mathcal{R}^{a}$ does not need to be a 1-1 function.

In order to formally capture the embodied perception ability of agents, a concept called the perceivable space is further introduced to refer to the internal and conceptually (mentally) possible domain of potentially recognizable objects available to an individual agent $a \in \mathcal{P}$, at the level of its strictly internal cognitive processes.

\section{Definition 5 (Perceivable Space)}

Each agent $a \in \mathcal{P}$ is assigned the perceivable space:

$$
\left.\mathcal{E}\right|_{a}=\left\{o_{i}^{a}: \exists i d_{j} \in \mathcal{E} \cdot \mathcal{R}^{a}\left(i d_{j}\right)=o_{i}^{a}\right\} .
$$

The perceivable space $\left.\mathcal{E}\right|_{a}$, assigned to a particular agent $a \in \mathcal{P}$, is understood as the maximal set of invariants' reflections that can ever be produced by this agent $a$ to map possible and external invariants into their internal representations, obviously by means of $\mathcal{R}^{a}$.

In order to be consistent with the aforementioned assumption that all language meaning originates from empirical data, in the forthcoming section each agent $a \in \mathcal{P}$ uses an actually activated, perceived space of invariants' internal reflections at each time point $t \in \mathcal{T}$. It is induced in its "mind" by and only by these invariants that have ever been captured by $\mathcal{R}^{a}$ during the system's runtime. What follows 
is that each $o_{j}^{a}=\mathcal{R}^{a}\left(i d_{i}\right)$ can be treated as an actual reflection of the invariant $i d_{i}$, embodied in an agent $a$, if and only if $i d_{i}$ has ever been both realized in the environment and reflected as an internal structure $\mathcal{R}^{a}\left(i d_{i}\right)$. Otherwise, it should be interpreted as and only as a formal name of a potentially recognizable invariant (perhaps physically available sometimes in the future). In order to reflect in further sections this aspect of language behaviour, at this point an additional concept of the perceived context, is introduced as follows:

\section{Definition 6 (Perceived Context)}

At each time point $t \in \mathcal{T}$ each agent $a \in \mathcal{P}$ is assumed to be equipped with an embodied representation of the perceived context $\mathcal{X}^{\mathcal{E}, a}(t)$, defined as follows:

$$
\mathcal{X}^{\mathcal{E}, a}(t) \subseteq\left\{o_{i}^{a}: \exists i d_{j} \in \mathcal{X}^{\mathcal{E}}(t) . \mathcal{R}^{a}\left(i d_{j}\right)=o_{i}^{a}\right\} .
$$

Next, the dynamic aspect of the population is captured in a momentary participation of certain agents in interactions. In order to describe interactions at particular time points $t \in \mathcal{T}$, a dedicated function $\mathcal{X}^{P}(t)$ will be used to yield a group of currently interacting agents (see Sec. III), as well as define their roles.

\section{Definition 7 (Interaction)}

The agents' interaction, realized at a given time point $t \in \mathcal{T}$, is defined by the interaction function as follows:

$$
\mathcal{X}^{P}: \mathcal{T} \rightarrow 2^{\mathcal{P}} \times 2^{\mathcal{P}},
$$

The value $\mathcal{X}^{P}(t)=\left(P_{S}, P_{H}\right)$ is a tuple, where $P_{S}$ and $P_{H}$ denote two disjoint sets of speaking and hearing agents, respectively.

Speakers are agents that play an active role in linguistic interaction. In particular, they analyse the available and perceivable context, select an object from it (topic), and utter the related topic's name. From a pragmatic point of view, the speaking agents' goal is to attract the attention of hearers to some invariant from the current context, using a linguistic clue in a form of a name utterance. Hearers, on the other hand, are passive learners. They analyse the available perceivable context and the uttered name, and try to infer intended meaning of the utterance. Thus, hearers focus on establishing an association between a word and an object that were utilised by a speaker.

\section{3. Agent}

Agents are the most fine-grained and autonomous parts of the system. They are both embodied members of the population, as well as situated in the environment. Each agent is equipped with a perceptual, operational and reasoning subsystem designed to carry out operations in the external world. However, in this paper a precise description of this subsystem is omitted. Namely, only a semantic infrastructure of agents needed to realize their linguistic behaviour is further exposed as a major part of the so-called agent's state (see [12]).

\section{Definition 8 (Agent State)}

For a particular agent $a \in \mathcal{P}$ and at a particular time point $t \in \mathcal{T}$, the state of the agent a is represented as the tuple $S_{t}^{a}$, given as follows:

$$
S_{t}^{a}=\left\langle O b_{t}^{a}, W_{t}^{a}, \mathcal{L}_{t}^{a}, \Gamma_{t}^{a}\right\rangle \in \Pi\left(S^{a}\right) .
$$

The following pragmatic interpretations and assumptions hold for this tuple:

- $\Pi\left(S^{a}\right)$ denotes the state space that is the space of all possible states in which the individual agent can stay.

- Ob $b_{t}^{a}$ denotes a set of objects that fulfill the requirement $\left.O b_{t}^{a} \subseteq \mathcal{E}\right|_{a}$. Each object in $O b_{t}^{a}$ represents a self contained invariant of the environment available to the agent's a perception and encapsulates the smallest indivisible entity available to its higher cognitive processes. For each agent a, each single object is explicitly identified by a unique and strictly internal reflections (identifier $o_{i}^{a}$ ). At a given time point $t \in \mathcal{T}$ the set of objects $O b_{t}^{a}$ is uniquely defined by all past interactions the agent a was involved in. In particular, the set of objects known for the agent a is defined as the following sum over all past perceivable contexts $O b_{t}^{a}=\bigcup_{\tau<t} \mathcal{X}^{\mathcal{E}, a}(\tau)$.

- $W_{t}^{a}$ denotes a set of words $W_{t}^{a} \in 2^{\mathcal{W}}$. Words are understood as external representations identified by the whole population as specialized communication signs. We further assume that there exists an infinite set of all possible words $\mathcal{W}=\left\{w_{1}, w_{2}, \ldots\right\}$ that can be identified by the population. Due to the populationwide availability of words we can assume that the utilised individual word identifiers $w_{i}$ are global and shared by agents. Nevertheless, at a particular time point $t \in \mathcal{T}$ an agent $a$ is familiar with and only with a certain subset $W_{t}^{a}$ of $\mathcal{W}$, i.e. $W_{t}^{a} \subset \mathcal{W}$.

- $\mathcal{L}_{t}^{a}$ denotes the lexicon mapping $\mathcal{L}_{t}^{a}: W_{t}^{a} \times O b_{t}^{a} \rightarrow$ $[0,1]$, embodied in the agent a. Such mapping $\mathcal{L}_{t}^{a}$ represents an actual correlation between objects $o_{i}$ and words $w_{j}$ by setting up an association strength for particular word-object pairs. Obviously, the higher the value of correlation the more convinced the agent a that the certain word is an adequate name for the object. In terms of linguistic pragmatics, the lexicon mapping constitutes one of the most important language-oriented cognitive structures, because it encapsulates the actual and momentary state of individually grounded language. For the sake of simplicity, we further assume that the space of all possible lexicon mappings from word-object pairs to values from $[0,1]$ available for the agent $\mathrm{a}$, will be denoted by $\Pi\left(\mathcal{L}^{a}\right)$ (consequently $\mathcal{L}_{t}^{a} \in \Pi\left(\mathcal{L}^{a}\right)$ ).

- $\Gamma_{t}^{a}$ denotes the word strength $\Gamma_{t}^{a}: W_{t}^{a} \rightarrow[0,1]$. In particular, in the LGM system each word $w_{i} \in W_{t}^{a}$ is associated with the agent's subjective notion of usability $\Gamma_{t}^{a}\left(w_{i}\right) \in[0,1]$ which denotes its individual estimation of popularity of the word over the population. 
The set of words that the individual uses is iteratively built up as new words are invented by speakers, whenever they lack a proper word for a particular given topic. New words are further incorporated by hearers whenever they receive unknown words for the first time. Dampening the word strength allows the agent to eliminate words that are not likely to be utilised by the population. Whereas, enforcing the word strength allows the agent to maintain words that are more intensively utilised by the population. Obviously, keeping track of words' popularity allows the agent to focus on the most popular linguistic terms (possible object names) and eliminate the least popular ones. For the sake of simplicity we further assume that the space of all possible word strength mappings will be denoted by $\Pi\left(\Gamma^{a}\right)$ (consequently $\Gamma_{t}^{a} \in \Pi\left(\Gamma^{a}\right)$ ).

To conclude, the agent's state $S_{t}^{a}$ is used to represent only the state of an embodied linguistic subsystem, given at a particular time point $t \in \mathcal{T}$.

Next, in order to formally refer to the language-related capabilities, the agent usage model is introduced. This model is used to define all computational means available for a particular agent $a$ to utilise its current state and realize relevant linguistic behaviour. Most importantly, the agent usage model defines an embodied computational method applied to produce and interpret current utterances. At the same time this model defines some attention oriented force that drives agents cognitive processes to certain objects of the environment. From a practical point of view, the agent usage model captures all methods applied by the agent to traverse the embodied lexicon and related word strengths. The agent usage model is defined subject to individual time points $t \in \mathcal{T}$, as follows:

\section{Definition 9 (Agent Usage Model)}

For each agent $a \in \mathcal{P}$ and at a given time point $t \in \mathcal{T}$, the agent's usage model is defined by the following tuple of three mappings:

$$
\left\langle\delta^{a}, \phi^{a}, \eta^{a}\right\rangle .
$$

For these mappings the following assumptions and interpretations, both pragmatic and formal, are assumed:

- $\delta^{a}$ denotes the interpretation function $\delta^{a}: W^{a} \times S_{t}^{a} \rightarrow$ $O b^{a}$. For a given state $S_{t}^{a}$ an agent $a \in \mathcal{P}$ is always able to interpret a particular external utterance $w_{j}$ by selecting the most adequate object $o_{i}^{a}$, using the current state of its lexicon $\mathcal{L}^{a}$. This way the actual lexicon state modulates the agent's interpretation scheme.

- $\phi^{a}$ denotes the production function $\phi^{a}: O b^{a} \times S_{t}^{a} \rightarrow$ $W^{a}$. In particular, for a given state $S_{t}^{a}$ an agent $a \in \mathcal{P}$ is always able to produce the value of $\left(\phi^{a}\right)$ as an external utterance $w_{j}$ by selecting the most adequate name for a given object $o_{i}^{a}$, using the current state of its lexicon $\mathcal{L}^{a}$. This way the actual lexicon state modulates the agent's production scheme.
- $\eta^{a}$ denotes the selection strategy $\eta^{a}: 2^{O b^{a}} / \emptyset \times S_{t}^{a} \rightarrow$ $\mathrm{Ob}^{a}$. Each interaction in the LGM (see Sec. II) is triggered by a speaker. It begins with an agent selecting a particular object from its sight as the topic of its utterance. Such a selection process models a particular intention that the agents are utilising (see [11]) and a particular strategy that depends on the actual task the system is faced with. For instance, the linguistic clue can be used by the agents to focus their individual attentions on a particular object from the environment, and further perform a certain mutual action upon it. In the related literature only the purely random selection of topic was investigated, where a speaker uniformly samples its current context in order to select the intended meaning of its utterance.

The remaining collection of mappings is called the agent update model. Its role is to define possible changes that each agent $a \in \mathcal{P}$ can undergo based on its current state and ongoing interactions. In particular, the agent update model characterises relevant changes of the lexicon, the word strengths and the set of words. Throughout the system's operation time, each agent $a \in \mathcal{P}$ is involved in many interactions, both as a speaker and hearer, and each interaction affects its internal state. For instance, the agent needs to update associations between a heard word and available objects, and the strength of the uttered word. In case an agent is a speaker and has no particular word for a particular object, it needs to additionally invent a novel word and introduce it to the lexicon. In general, the agent update model defines the embodied method to update the state of the agent. Formally, this model is defined, too, subject to individual time points $t \in \mathcal{T}$, as follows:

\section{Definition 10 (Agent Update Model)}

For each agent $a \in \mathcal{P}$ and at a given time point $t \in \mathcal{T}$, the agent's update model is defined by the following tuple of three mappings:

$$
\left\langle\theta^{a}, \vartheta^{a}, \kappa^{a}\right\rangle .
$$

For these mappings the following assumptions and interpretations, both pragmatic and formal, are assumed:

- $\theta^{a}$ denotes the lexicon update $\theta^{a}: W^{a} \times 2^{O b^{a}} \times S_{t}^{a} \rightarrow$ $\Pi\left(\mathcal{L}^{a}\right)$. Learning from co-occurrence between words and objects (cross-situational learning) implies that after each interaction the hearer updates its lexicon $\mathcal{L}^{a}$ by modifying related $\left(o_{i}, w_{j}\right)$ correlations.

- $\vartheta^{a}$ denotes the word strength update $\vartheta^{a}: W^{a} \times 2^{O b^{a}} \times$ $S_{t}^{a} \rightarrow \Pi\left(\Gamma^{a}\right)$. At the individual agent level the interpreted meaning and the heard word are regarded as the most probable ones. Based on the word strength update function, the agent's subjective notion of usability of the heard word $\Gamma^{a}$ is modified. In particular, the update function defines how the linguistic clue (in a form of utterance produced by the speaker) 
should affect and influence learner's naming convention through proper modification of related association structures in the lexicon.

- $\kappa^{a}$ denotes the word creation update $\kappa^{a}: W^{a} \times 2^{O b^{a}} \times$ $S_{t}^{a} \rightarrow 2^{\mathcal{W}}$. The word creation defines the means for inventing and adopting new names for unnamed objects. Whenever an agent is playing the role of the speaker, it is required to name a particular object from the perceivable context. In particular, if the agent has no name for a selected object $o^{t}$, a need develops to introduce a novel word. The agent tends to select a particular word $w \in \mathcal{W}$ from the set of all available words and creates all needed association in the lexicon. On the other hand, whenever an agent registers an unheard word it also needs to be able to introduce this word into its lexicon system. Such behaviour is realised by the word creation update function.

The whole agent's system can now be described by means of the following four rules:

- the production rule yielding a name for an object,

- the interpretation rule yielding an object for a name,

- the word creation rule inventing/adopting new names for objects,

- the update rules updating the association scores.

In consequence, at a general level the complete agent model used in this research can be summarized as follows:

\section{Definition 11 (Agent Model)}

For a given agent $a \in \mathcal{P}$ and a time point $t \in \mathcal{T}$, the agent model is represented by the following tuple:

$$
\left\langle O b^{a}, W^{a}, \Gamma^{a}, \mathcal{L}^{a}, \phi^{a}, \eta^{a}, \delta^{a}, \theta^{a}, \vartheta^{a}, \kappa^{a}\right\rangle,
$$

provided that previously accepted assumptions and interpretations, both pragmatic and formal, hold.

\section{4. System}

It follows from the above that the dynamic character of our system results from the following three basic processes:

- the world dynamics involved in the process of selection of context objects,

- the population dynamics involved in the process of selection of interaction participants, and

- the topic selection resulting from the process of selection of topic from the context.

Finally, the notion of a system state $S_{t}$, understood as the state of the entire multi-agent system, is captured by the following definition:

\section{Definition 12 (System's State)}

For each $t \in \mathcal{T}$ the system state $S_{t}$ at the time point $t$ is represented by the following tuple:

$$
S_{t}=\left\langle\mathcal{E}, \mathcal{P}, \mathcal{X}^{\mathcal{E}}, \mathcal{X}^{P}\right\rangle
$$

where $\mathcal{E}, \mathcal{P}, \mathcal{X}^{\mathcal{E}}, \mathcal{X}^{P}$ denote the environment, the population, the context and the interaction, respectively.
This definition completes the technical presentation of our system's constituents.

\section{META-MODEL INSTANTIATIONS}

The extensive literature studies, including the most recent summaries in $[20,22]$, show that despite of its popularity the LGM has been investigated in a rather limited set of basic settings. This remark applies to formal models utilised by other authors (if at all specified), too. As such, previous limited approaches, although valuable and influential, have not provided an expected general perspective. In this context, the presented meta-model is a functional extension of many original formulations of LGMs, known from the related literature. In particular, it captures the basic settings as well as additional interesting features worth being considered in language alignment games.

In consequence, in our further research we try to follow the basic settings of LGM (where possible), and introduce some extensions to possible interaction patterns. At the same time, we keep maintaining the substantial coherence with the basic LGM to relate to it in our further analysis of language behaviour realized within alignment processes with multiparticipant interaction patterns. In particular, we explore how the changing interaction conditions affect the ability of the population to reach coherent naming conventions. Multiple dimensions of modifications of the interaction conditions are briefly suggested as follows.

\section{1. Perception characteristics}

Perception abilities of artificial systems are crucial to any form of language behaviour and other cognitive processes. In general, it is the perception ability that defines the actual content and shape of perceivable spaces available to higher level cognitive processing. These obvious inter-wind characteristics of the perception and cognition defines what information in what form is available to an individual.

\section{1. 1. Population vs. environment}

Depending on a particular relation between the structure of the environment and a particular structure of system's perception abilities we distinguish two possible settings: idealised with perfect alignment between perception and external world, and non-idealised with certain misalignments between perception and external world. Assuming further the aforementioned invariant-oriented structure of the environment (see Def. 1) we introduce both settings.

In the idealised settings the system is able to maintain a one-to-one mapping between the invariants available in the external world $\mathcal{E}$ and the internal reflections available to the individual $\left.\mathcal{E}\right|_{a}$. In particular, all agents are capable of a nearly direct perception of the environment. 


\section{Definition 13 (Idealised Perception Settings)}

The idealised perception settings hold in the system iff for every agent $a \in \mathcal{P}$ the following formula holds:

$$
\left.\forall i d_{j} \in \mathcal{E} \exists o_{i}^{a} \in \mathcal{E}\right|_{a} . \mathcal{R}^{a}\left(i d_{j}\right)=o_{i}^{a} \wedge\left\|\left.\mathcal{E}\right|_{a}\right\|=\|\mathcal{E}\| .
$$

In the non-idealised settings the system is unable to maintain a consistent one-to-one mapping between the invariants available in the external world $\mathcal{E}$ and the internal reflections $\left.\mathcal{E}\right|_{a}$. In particular, the system might not be able to identify and/or distinguish between all invariants $i d_{i} \in \mathcal{E}$, as such leaving certain parts of possible space unavailable to the system. Such a setting can also be regarded as a particular systematic error of the perception mechanism that prevents the idealised case. Assuming the aforementioned definition of the idealised settings (see Def. 13) we can generally define the non-idealised settings as follows:

\section{Definition 14 (Non-Idealised Perception Settings)}

The non-idealised perception settings hold in the system iff for every agent $a \in \mathcal{P}$ the following formula holds:

$$
\left\|\left.\mathcal{E}\right|_{a}\right\| \neq\|\mathcal{E}\| \text {. }
$$

\section{1. 2. Population homogeneity}

In the presented research we are particularly focused on the interaction between individuals that the system is comprised of. As such it is important to distinguish an additional perspective that relates to a particular relation between the perception capabilities of individuals that form the entire system. We distinguish two possible settings: homogeneous, where all agents are equipped with coherent perception abilities, and heterogeneous, where the individual perception abilities can differ between the agents.

In the homogeneous settings all agents are able to perceive and distinguish the same set of invariants from the environment (see Def. 15).

\section{Definition 15 (Homogeneous Perception Settings)}

The homogeneous perception settings hold in the system iff for each pair of agents $a, A \in \mathcal{P}$, such that $a \neq A$, the following formula holds:

$$
\begin{aligned}
& \left(\left.\left.\forall o_{i}^{a} \in \mathcal{E}\right|_{a} \exists i d_{j} \in \mathcal{E} \exists o_{i}^{A} \in \mathcal{E}\right|_{A} \cdot \mathcal{R}^{a}\left(i d_{j}\right)=o_{i}^{a} \wedge \mathcal{R}^{A}\left(i d_{j}\right)=o_{i}^{A}\right) \\
& \quad \wedge\left\|\left.\mathcal{E}\right|_{a}\right\|=\left\|\left.\mathcal{E}\right|_{A}\right\| \neq 0 .
\end{aligned}
$$

Note: It should be remembered that $\mathcal{R}^{a}$ is assumed to be a function.

In the above setting there exists a one-to-one mapping between individual perceivable spaces. As opposed to the idealised settings there is no requirement that all available invariants are directly available to individual agents. For instance, due to a given set of equipped sensors the individual units are only capable of registering a respective set of sensory data. Moreover, due to the physical limitations individual agents might not be able to discriminate between a certain group of invariants present in the environment. Consequently, a certain part of the environment can be entirely unavailable to the system, i.e. to all individual units, whereas the available part is perceivable by the system in a coherent manner, i.e. isomorphically by individual units.

In the heterogeneous settings individual perceptions can differ among the agents. Opposite to the homogeneous settings, it is impossible to split the perception into two sets of unavailable and isomorphic invariants. Consequently, a certain part of the environment is perceived differently by the system.

\section{Definition 16 (Heterogeneous Perception Settings)}

Heterogenous perception settings hold in the system iff the homogeneous perception settings do not hold.

Remark: Obviously, the heterogeneous perception settings cover a diverse set of cases, where the homogeneity does not hold.

In such a setting, it is impossible to establish a one-toone mapping between individual perceivable spaces, as at least a single agent is characterised with different abilities. For instance, the system might comprise of different types of individuals (perhaps physically), that operate together in a common environment.

\section{1. 3. Population vs. context}

The underlying perception processes, i.e. how a group of agents perceives the external world, directly affect the alignment processes. As aforementioned, a particular context (see Def. 6) serves as the only source of meaning, i.e. a source that is objectively existing and possibly shared. There are many possible implementations of a group perception, for instance the interacting agents can perceive and share the entire context, can perceive only a shared part of the context, or can differently perceive some parts of the context, or alternatively perceive different parts of the context.

We distinguish three possible practical realisations of the interaction scene, i.e. a particular relation between individual perceptions (realised by agents involved in the interaction) of the current context $\mathcal{X}^{\mathcal{E}}$ : joint attention scene, common context scene, and disjoint scene.

In the joint attention scene a group of agents engaged in an interaction perceives and shares the entire context. All interacting agents register the same set of invariants, but through different internal representations. In particular, a joint attention scene defines a situation in which all interacting agents perceive exactly the same context, i.e. the same set of distinguishable invariants.

\section{Definition 17 (Joint Attention Scene)}

The joint attention scene is established at a given time point $t \in \mathcal{T}$ iff for each pair of interacting agents $a, A \in P$ 
$(P \subset \mathcal{P}$ and $P \neq \emptyset$ ), so that $a \neq A$, the following formula holds:

$$
\left(\forall \mathcal{R}^{a}\left(i d_{j}\right) \in \mathcal{X}^{\mathcal{E}, a}(t) . i d_{j} \in \mathcal{X}^{\mathcal{E}}(t) \Rightarrow \mathcal{R}^{A}\left(i d_{j}\right) \in \mathcal{X}^{\mathcal{E}, A}(t)\right)
$$$$
\wedge\left\|\mathcal{X}^{\mathcal{E}, a}(t)\right\|=\left\|\mathcal{X}^{\mathcal{E}, A}(t)\right\| \neq 0
$$

In the idealised settings joint attention scene implies that the agents are able to perceive the entire context. In the nonidealised settings joint attention scene implies that the agents perceive the same part of the context that consists of distinguishable invariants. In particular, if the context consists of only distinguishable invariants, then the interacting agents perceive the entire context or its subset. However, if the context consists of both distinguishable and indistinguishable invariants, then the interacting agents perceive only the distinguishable part of the context.

It is rarely the case when agents involved in an interaction are able to perceive the same exact contexts. For instance, due to the inherent autonomy of individuals their attention can be oriented towards a particular type of objects from the current context, or a particular sub-part of the current context. In particular, a common scene determines a situation in which individual agents are able to focus on different aspects of the available environment, whilst still maintaining a certain common part (common ground of the context). In order to capture such a situation we further introduce the notion of a common context scene.

\section{Definition 18 (Common Context Scene)}

The common context scene is established at a given time point $t \in \mathcal{T}$ iff for every pair of interacting agents $a, A \in P$ $(P \subset \mathcal{P}$ and $P \neq \emptyset$ ), so that $a \neq A$, the following formula holds

$$
\begin{aligned}
\exists i d_{j} & \in \mathcal{X}^{\mathcal{E}}(t) \exists \mathcal{R}^{a}\left(i d_{j}\right) \in \mathcal{X}^{\mathcal{E}, a}(t) . \\
\mathcal{R}^{A}\left(i d_{j}\right) & \in \mathcal{X}^{\mathcal{E}, A}(t) .
\end{aligned}
$$

Due to the inherent heterogeneous nature of individuals some invariants in the context can be indistinguishable. Opposite to the case when all agents involved in an interaction are able to perceive the same context, here all agents perceive only a shared subset of invariants from the available context - a common part of the context.

In the idealised settings, as the agents are able to identify and distinguish between all available invariants, a common context scene involves a situation in which certain agents are unable to observe certain parts of the context. In the nonidealised or heterogeneous settings the common subset of invariants can be perceived differently, due to the inherent inability to discriminate between all invariants. From a practical stance, such a situation represents a case where agents are able to establish a common ground in an interaction, but this common ground may slightly differ between agents. Obviously, such a restriction guarantees that interacting agents are able to distinguish a non-empty part of the context.

In the least restrictive situation the agents involved in an interaction can perceive entirely different subsets of invariants and/or these invariants can be additionally indistinguishable by individual agents.

\section{Definition 19 (Disjoint Context Scene)}

The disjoint attention scene is established at a given time point $t \in \mathcal{T}$ iff there exists a pair of interacting agents $a, A \in P(P \subset \mathcal{P}$ and $P \neq \emptyset)$, so that $a \neq A$, for which the following formula holds:

$$
\begin{aligned}
\exists i d_{j} & \in \mathcal{X}^{\mathcal{E}}(t) . \\
\mathcal{R}^{a}\left(i d_{j}\right) & \in \mathcal{X}^{\mathcal{E}, a}(t) \Rightarrow \mathcal{R}^{A}\left(i d_{j}\right) \notin \mathcal{X}^{\mathcal{E}, A}(t) .
\end{aligned}
$$

The disjoint context scene represents a case where there is no common ground between all of the interacting agents. In such a case, the current context cannot serve as an existing objectively shared source of meaning, as it is actually not shared. Consequently, as there is no source to anchor the meaning of a given label to, such a situation cannot guarantee a successful formulation of a shared naming convention.

\section{1. 4. Knowledge base}

The additional aspect of agent's perception characteristics involve the internal organisation of gathered experience. In the basic approach (see [6] [5]) a static and a fixed set of perceivable objects is assumed and this is common practice in the LGM-based research. It represents a situation when the agents start with a predefined set of known and predefined objects, and no other object can enter the perceivable space of an individual. In this paper we extend the basic approach by allowing the agent to gradually build up its set of known objects $O b_{t}^{A}$. In particular, as the agent encounters a new distinguishable invariant in the environment, it stores its representation and updates its private databases.

In practice, the set of objects available in the environment $\mathcal{E}$ is not directly available to the agent, certainly not during the early stages of the system's operation $\left(\left.O b_{t}^{A} \subset \mathcal{E}\right|_{A}\right)$. In consequence, early interactions cannot affect the entire scope of objects (all objects available in the environment $\left.\left.\mathcal{E}\right|_{A}\right)$, but rather affect only the set of known objects $\left(O b_{t}^{A}\right)$. Such an extension has a significant pragmatic value, as it is unrealistic to assume that designers embodying a multiagent system in a versatile and unknown environment are able to explicitly identify all possible objects that can be encountered by the agents beforehand (at the design time). It is significantly easier for the system to gradually (during its operation) identify novel objects and further introduce them into their linguistic structures. 


\section{2. Lexicon processing}

We further assume the well established mechanism of interpretation and production (see [6] [5]). The interpretation scheme is rather straight-forward, as for a given word $w_{i} \in W_{t}^{a}$ the interpretation function $\delta^{a}$ determines the object $o_{j}^{a}$ with the maximum weight $\left(\delta^{a}\left(w_{i}, t\right)=\right.$ $\operatorname{argmax}_{o_{k}^{a} \in \mathcal{E} \mid{ }_{a}} \mathcal{L}_{t}^{a}\left(o_{k}^{a}, w_{i}\right)$ ), and thus interprets $w_{i}$ as referring to $o_{j}^{a}$. On the other hand, the production scheme assumes that the speaker before uttering a name evaluates its subjective reflection of the population by considering the strength $\Gamma^{a}$ of each possible word $w_{i}$. As such, for a given object $o_{j}^{a}$ the production function $\phi_{t}^{a}\left(o_{j}^{a}\right)$ determines the word $w_{i} \in W^{a}$ having the highest usability from all the words that the agent is able to interpret as referring to the object $o_{j}^{a}\left(\phi_{t}^{a}\left(o_{j}^{a}, t\right)=\operatorname{argmax}_{\left\{w_{k} \in W^{a} \wedge \delta^{a}\left(w_{k}, t\right)=o_{j}^{a}\right\}} \Gamma_{t}^{a}\left(w_{k}\right)\right)$, and thus names $o_{j}^{a}$ using $w_{i}$.

In this research we incorporate ICSL (see [6]) and INT (see [12]) strategies of lexicon update (alignment mechanism) and word strength update definition from the approach defined in [6]. Both of the assumed approaches are based on the associationistic lexicon approach with weighted wordobject pairings $\mathcal{L}_{t}^{a}\left(o_{k}^{a}, w_{i}\right)$ where each word is additionally weighted by its strength $\Gamma^{a}$. Moreover, they both represent the so-called dampening strategies, where at each iteration the agent not only enforces the associations between the heard word and currently perceived objects (in the current context), whilst still dampening the associations between the heard word and all of the currently unavailable objects (outside of the current context).

The idea behind the ICSL strategy is that the learning procedure maintains the association values within a restricted interval, i.e. $\mathcal{L}_{t}^{a}\left(o_{k}^{a}, w_{i}\right) \in[0,1]$ and $\Gamma^{a}\left(w_{i}\right) \in[0,1]$. Moreover, at each interaction episode the strength of the update procedure is modulated by the value of the current cumulative sum of strengths distributed among the heard word $o^{t}$ and all available context objects $\mathcal{X}^{\mathcal{E}, a}(t)$. Further, the underlying goal is to increase the cumulative sum of consistent objects by a given update strength factor and distribute it among context objects according to their current strengths. Consequently, the uncertainty involved in the size of the context is directly incorporated, as the increase value is distributed among all objects within the context. Moreover, the strengths related to objects outside the context are decreased, i.e. to decrease the cumulative strength of inconsistent objects. The important property of the ICSL strategy is adaptivity. In particular, as the association values are bounded $([0,1])$ the entire process allows the individual agents to relatively swiftly change the state of the lexicon.

On the other hand, the INT strategy represents a basic dampening approach, where a strict and constant dampening strength is assumed. However, the INT strategy is additionally decreasing the associations between concurring words. In particular, the association between the heard word and all consistent objects is enforced, the association between the heard word and inconsistent objects is dampened, and association between the interpretation of the heard word (object) outside of the context is additionally decreased. As such, the strategy focuses on maintaining the number of concurring words at a minimal level.

Additionally, we incorporate two word creation schemes, i.e. novel words constructed by the speaker can either be unique or non-unique. The difference between the two lays in the fact that the former always involves that a new word (its form) includes the agent's individual unique identifier. In essence, the latter allows different agents to create identical words (independently of each other), and associate them (initially) with different objects in the external world. The non-unique approach is characterised by significant levels of homonymy, in particular in the early stages of alignment, whereas in the unique approach the invented words are unique and as such we assure that no two invented words are identical. Consequently, there is less homonymy within the system, but possibly more synonymy as there are multiple different words associated with a single object (especially in the early stage of development) by multiple agents. Nevertheless, from a pragmatic stance a particular practical implementation depends solely on the system's inherent ability to create unique linguistic forms.

Additionally, all current models of the language game assumed a single-stage pair-wise interaction. In particular, all involved a basic interaction $\mathcal{X}^{P} \in \mathcal{P} \times \mathcal{P}$, where a pair of agents (one acting as a hearer and the other as a speaker) is involved in a simple game. Such a game always involves only a single act of communication, hence the name singlestage, and after the interaction the process continues, i.e. new context is generated, a new pair of agents is determined, etc.

We can note that the study of multi-stage and multiparticipant interactions is neglected in the literature despite the fact that the pair-wise single-stage interaction represents an idealised model that in practice requires additional coordination mechanisms. In particular, it is crucial to maintain an isolated pair of agents and limit it to a single speaker and a single hearer, whereas in practice a multi-stage and multiparticipant interaction seems to be more frequent and more convenient to use.

However, in real robotic systems the assumption of pairwise interaction is hard to achieve and rather impractical, and in actual settings it is significantly more frequent and certainly more convenient to introduce a multiple participants model. For example, a basic and explicit broadcast scheme could be easily implemented allowing agents to communicate in a less restrictive multi-participant scheme. As such, we allow our system to be more flexible in terms of possible interaction patterns. Additionally, in real embodied multiagent systems we cannot guarantee that each interaction would involve only a pair of agents without externally enforcing such settings. For instance, as the agents are utilising a form of linguistic communication, it is highly probable 
that nearby agents would also engage in a particular interplay. It should be underlined that from the point of view of the LGM these settings again fall outside of the basic model.

\section{3. Interaction Patterns}

The idea behind the language game is that through a series of routine interactions the agents can align their lexicons reaching a coherent state of the entire population. The interaction is realised through a sequence of individual episodes in which agents utilise association-based lexicon $\mathcal{L}$. Finally, the linguistic communication is the only form of communication available to the agents. As such, there are no additional signalling mechanisms, nor any form of telepathy. Consequently, the linguistic interplay between the agents is the only opportunity for a hearer to gain insights about the lexicon utilised by the speaker.

In this paper we investigate a multi-participant extensions to the previously mentioned basic pair-wise interaction pattern incorporated in the LGM. In particular, in a given state of the external environment (called the context) we investigate settings with multiple speakers and multiple hearers involved in a single interaction, as such allowing the system to be more flexible in terms of defined interaction.
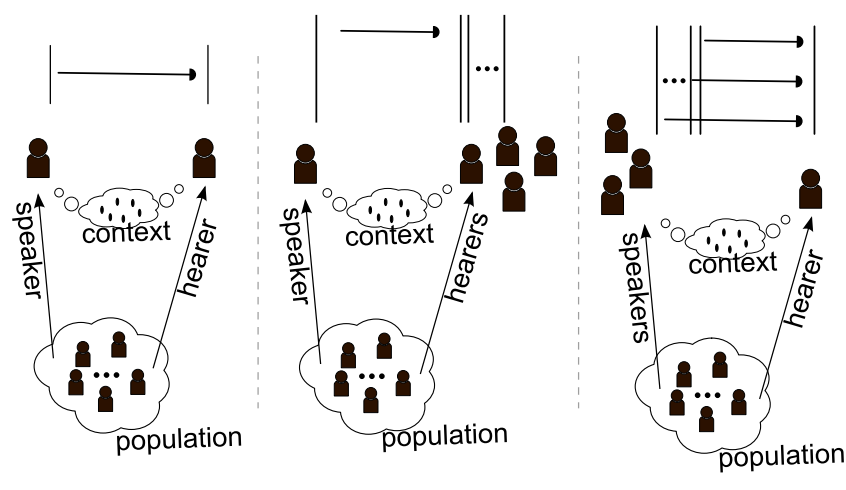

Fig. 2. Interaction Patterns - pair-wise, multi-hearers and multispeakers

Obviously, it becomes crucial to study how the above multi-agent game influences the alignment processes. It should be underlined that we do not modify the underlying behaviour of an individual agent. In essence, we solely focus on the behaviour of the basic model (with extended interaction settings) and treat individual communication acts as separate, though bounded by the current context and interacting agents.

Below we introduce two analysed modes of interaction: a two-participant mode in a pair-wise pattern, and a multiparticipant mode in multi-speakers and multi-hearers patterns.

\section{3. 1. Pair-wise interaction pattern}

In the pair-wise interaction pattern the interplay between the agents is governed by a strict pair-wise interaction scheme. At each of such episodes two agents are randomly selected from the population, one acting as a speaker, and the other acting as a hearer. Both agents are embodied and situated in the same state of the environment, thus establishing a joint attention scene. In such setting the speaker tries to draw attention of the hearer to a particular element in the shared scene (called topic), using a linguistic clue (called $u t$ terance). As a result, the hearer is trying to correctly relate the linguistic utterance to a particular element of the current state of the environment.

\section{Definition 20 (Pair-wise Interaction)}

In the pair-wise interaction pattern, for each time point $t \in \mathcal{T}$, the value of the interaction function $\mathcal{X}^{P}(t)=\left(P_{S}, P_{H}\right)$ fulfills the following condition:

$$
\left\|P_{S}\right\|=1 \wedge\left\|P_{H}\right\|=1
$$

In this type of interaction only a single speaker and a single hearer are allowed.

\section{3. 2. Multi-speaker interaction pattern}

In the multi-speaker interaction pattern multiple agents engage themselves in communication. Namely, in a given state of the environment (called common context) multiple agents, at least 3, meet and interact. Among them one and only one acts as a hearer (see Def. 21).

\section{Definition 21 (Multi-speaker Interaction)}

In the multi-speaker interaction pattern, for each time point $t \in \mathcal{T}$, the value of the interaction function $\mathcal{X}^{P}(t)=\left(P_{S}, P_{H}\right)$ fulfills the following condition:

$$
\left\|P_{S}\right\|>1 \wedge\left\|P_{H}\right\|=1
$$

In this type of interaction multiple agents participate but exactly one acts as a hearer.

At each interaction multiple agents are selected from the population (as of $\mathcal{X}^{P}(t)$ ). One of the selected agents acts as a hearer, whilst others are speakers. All interacting agents are situated in the same state of the environment, thus establishing a joint attention scene called the shared state of local surroundings. In such a setting each speaker utters a name of a particular invariant in the external world and using such linguistic clue tries to draw attention of the hearer. As a result, the hearer registers multiple words (even some might be repeating), all uttered in the same context, and tries to correctly relate the linguistic utterances to certain elements of the current state of the environment. 


\section{3. 3. Multi-hearer interaction pattern}

In the multi-hearer interaction pattern multiple agents engage in communication. In a given state of the external environment (common context) multiple agents, at least 3 , meet and interact. In particular, a single agent acts as a speaker and multiple agents act as hearers (see Def. 21).

\section{Definition 22 (Multi-hearer Interaction)}

In the multi-hearer interaction pattern, for each time point $t \in \mathcal{T}$, the value of the interaction function $\mathcal{X}^{P}(t)=\left(P_{S}, P_{H}\right)$ fulfills the following condition:

$$
\left\|P_{S}\right\|=1 \wedge\left\|P_{H}\right\|>1 .
$$

In this type of interaction multiple agents participate but exactly one acts as a speaker.

At each interaction, multiple agents are selected from the population (as of $\mathcal{X}^{P}(t)$ ). One of the selected agents acts as a speaker, whilst all other as hearers. All interacting agents are situated in the same state of the environment, thus establishing a joint attention scene. In such a setting, a single speaker utters a name of a particular invariant in the environment - intended topic. In particular, using a linguistic clue, the speaker tries to draw attention of all hearers to a particular invariant in the environment. As a result, all hearers register a single word, all uttered in the same context, and all try to correctly relate the linguistic utterance to a certain element of the current state of the environment.

\section{SIMULATION}

In order to investigate the influence of different interaction patterns on the behaviour of the multi-agent systems we perform an exhaustive series of simulations. All experiments share a common framework (see Sec. II) and assume a finite, static set of objects and agents, and all are restricted to a shared context setting $\left(o^{t} \in \bigcup_{a \in \mathcal{P}} \mathcal{X}^{\mathcal{E}, a}(t)\right)$. We investigate a number of simulation settings, but due to the space limitations we only focus on the general properties of the system and present the obtained results as an exemplification of the observed system's behaviour.

Let us assume the following experiment settings for all of the underlying simulations (baseline settings):

- population: $\|\mathcal{P}\|=10$,

- environment: $\|\mathcal{E}\|=\left\|\left.\mathcal{E}\right|_{a}\right\|=10$, (for all $a \in \mathcal{P}$ ),

- context: $\left\|\mathcal{X}^{\mathcal{E}}(t)\right\|=\left\|\mathcal{X}^{\mathcal{E}, a}(t)\right\|=2$, (for all $a \in \mathcal{P}$ ),

- learning strategy: ICSL / INT ${ }^{3}$,

- selection strategy: uniform,

- word creation: with / without unique word creation ${ }^{3}$.
In such a framework we further investigate the behaviour of alignment processes in disparate interaction models:

1. pair-wise model: $\left\|P_{S}\right\|=1$ and $\left\|P_{H}\right\|=1$,

2. multi-hearers model: $\left\|P_{S}\right\|=1$ and $\left\|P_{H}\right\| \in \overline{2,5}$,

3. multi-speakers model: $\left\|P_{S}\right\| \in \overline{2,5}$ and $\left\|P_{H}\right\|=1$.

It should be noted that all of the presented graphs are an average over multiple consecutive runs (at least 50) and as such are a good representation of the observed dynamic behaviour of the system. Wherever possible we not only provide the average over runs but present more informative boxplots.

In order to formulate differences in the dynamics of the alignment processes, we identify two major axes of comparison: coherence and word statistics, and focus on the evolution of language in the assumed multi-agent system. We study the behaviour of the system based on three measures: language coherence rate, average number of used words and the overall number of words.

Language coherence rate (LC) resembles the spread of the naming convention among the entire population and reflects the coherence of names among all existing objects. As such, we introduce language coherence $\mu_{L C}$, as the probability that two randomly selected agents assign the same name for a randomly selected object from the environment, as follows ${ }^{4}$ :

\section{Definition 23 (Coherence Measure)}

$$
\begin{aligned}
& \mu_{L C}= \\
= & \left\langle\mathcal{I}\left[\delta^{A}\left(\phi^{a}\left(\mathcal{R}^{a}\left(i d_{k}\right), \mathcal{L}^{a}\right), \mathcal{L}^{A}\right)=\mathcal{R}^{A}\left(i d_{k}\right)\right]\right\rangle_{a, A \in \mathcal{P}, i d_{k} \in \mathcal{E}} .
\end{aligned}
$$

The lowest possible coherence, i.e. $\mu_{L C}=0$, reflects a state of no language coherence in the system, as there are no two agents that use the same name for any of the objects. The highest possible coherence, i.e. $\mu_{L C}=1$, represents the state of full coherence, where all agents share the same naming conventions. It should be noted that in the assumed settings the coherent state is absorbing the system, as from this point all of the utterances are consistent with the observed context, and without any external disturbance all of the strongest associations remain strongest.

In order to analyse the characteristics of the emergent language we keep track of the number of used words $\mu_{U W}$ (UW), and keep track of the total number of all invented words $\mu_{T W}$ (TW), defined as follows:

\footnotetext{
${ }^{3}$ We introduce multiple variants in order to underline that the obtained results represent a general behaviour of the alignment processes. In particular, we do not intend to provide a detailed comparison of the introduced variants, as we solely focus on the effects of different interaction patterns.

${ }^{4}\langle X\rangle_{Y}$ defines the average value of $X$ over $Y$.
} 


\section{Definition 24 (Number of Used Words)}

$$
\begin{aligned}
& \mu_{U W}= \\
= & \left\langle\left\|\left\{w \in W^{a}: \exists o_{a}^{i} \in O b^{a} \cdot \mathcal{L}^{a}\left(w, o_{a}^{i}\right)>0\right\}\right\|\right\rangle_{a \in \mathcal{P}} .
\end{aligned}
$$

\section{Definition 25 (Number of Words)}

$$
\mu_{T W}=\left\langle\left\|W^{a}\right\|\right\rangle_{a \in \mathcal{P}} .
$$

The number of used words is calculated over all agents as the average number of positively associated words, and it resembles the stability of current associations. As the optimal communication system has one-to-one mappings between words and objects, i.e. the same number of used words as the number of existing objects, any deviation from this proportion reflects a potentially unstable situation, as miscommunication might occur. However, the total number of words defines the average number of words known by an agent.

\section{ANALYSIS}

In order to study the dynamics of the alignment processes, we use the whole set of distinguished measures that allow us to track the significant changes that trigger within the system.

\section{1. Pair-wise Pattern - single speaker and single hearer}

After analysing the obtained simulation results (see Fig. 3 and Fig. 5), we can note that the alignment process can be decomposed into a four stage process. However, there is no sharp transition between the distinguished stages, i.e. a rather smooth transition. In particular, depending on the type of word creation pattern, i.e. unique or non-unique, the system either directly enters the first stage (unique) or enters a certain pre-early stage (non-unique). In the latter case, first iterations result in a sudden increase of coherence, due to the coincidental (in terms of their relation to the external word) creation of identical names, which relatively quickly returns to initially low values. Nevertheless, despite this early difference in the character of dynamic evolution of the alignment processes, once in the initial stage both undergo similar changes, i.e. in respect to proper scaling.

The first stage involves the word creation phase. In particular, the initial words that enter the system are rapidly aligned, i.e. by agents that due to a particular realisation of the interaction process $\mathcal{X}^{P}$ managed to engage in an interaction. Consequently, a somewhat coherent subgroups form within the population. It should be noted that these subgroups are strictly focused and relate only to a particular form-meaning mappings, and certainly not to the entire lexicon (over all available objects). In essence, the agreement upon a certain naming convention within the entire population, is relatively low, as the population managed to develop only small and local similarities between the lexicons. Nevertheless, despite being small they sum up to relatively high values, as compared to the initial coherence rate $(0)$, hence the early significant increase of the coherence rate. Moreover, as new words are intensively introduced into the system we can indicate the increase of the number of words in general and the number of used words. Finally, the first stage ends when the agents manage to developed enough words, i.e, each agent is able to name all of the objects it encountered in the external word. We note that the maximal number of used words for the given settings is about 20-21 (36-37) and it is reached after 1300-1500 (800-1000) iterations, roughly 100 iterations per agent and 45 iterations as a speaker, with unique words (with non-unique words).

The second stage is a testing stage, where the agents test all of the current associations. This behaviour dominates over the population, i.e. agents reinforce the consistent formmeaning parings and dampen the inconsistent ones. In particular, the initial associations are contrasted with associations developed by other agents. Consequently, on average a single form-meaning pair is rather unpopular within the population (small coherence rate, below 0.4). Nevertheless, as the lexicons fill-up with form-meaning pairings the agents start to compete with each other, in particular the naming conventions that they represent. At that stage (roughly after 1000 interactions, i.e. on average 45 interactions per agent and roughly 19 interactions as a hearer and a speaker), the average number of used words reaches its peak value and the coherence dynamics reaches a point of inflection. In particular, as there are multiple words competing for the same object, then after an interaction between two agents utilising different conventions the hearer is forced to decrease its individual certainty towards a particular word it used and increase the certainty towards the word uttered by the speaker. Moreover, as the former dominated the lexicon after the lexicon update procedure its strength is eventually decreased. Certainly such a decrease is only up to a certain point, as in the place of one word there are other words that compete with it.

On the other hand, the early languages consist of many words (the average number of used words is roughly twice the number of objects, in the case of unique words, and roughly four times the number of objects, in the case of non-unique words), i.e. after enough iterations the agents on average managed to interact with all other agents in nearly all possible settings of the external world. Consequently, the agents are able to adopt most of the words present in the system, i.e. early increase of the average number of used and the total number of words is nearly identical.

The third stage is the elimination stage, where the unpopular associations (due to a particular realisation of the interaction and context process) are eliminated by the pop- 

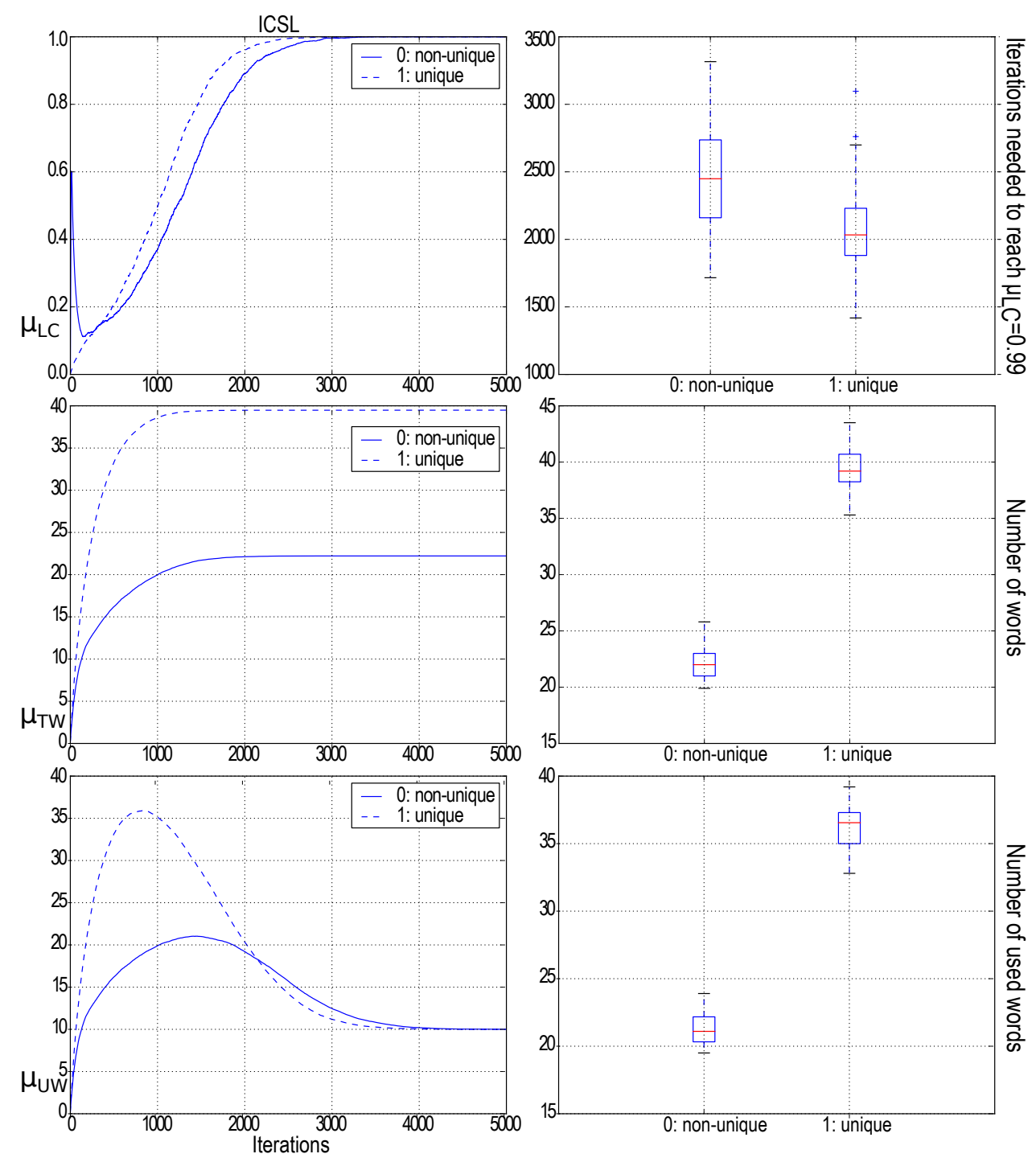

Fig. 3. Single-stage with single participants (ICSL) - coherence dynamics (top row), number of words (middle row) and number of used words (bottom row)

ulation, as there is no pressure to reinforce such associations. In particular, these unpopular conventions include form-meaning pairings that are utilised only by a small group of agents and directly influence the decrease in the number of used words, as the unpopular associations die out (strengthening the language and increasing the coherence). Next, the system is again faced with the problem of minority, i.e. the process of alignment slows down. In particular, we note that as the phenomena with relatively small probability need to trigger, there is a large number of iterations that do not directly result in a significant change in the coherence rate, i.e. we can note that an individual association in a population makes only for a tiny fraction of the coherence $-\left(N \cdot\left(0.5 \cdot\|\mathcal{E}\| \cdot\left(\mathcal{P}^{2}-\mathcal{P}\right)\right)^{-1}\right)$, where $N$ is the number of agents already using a particular convention). Nevertheless, during that stage of system's dynamics a particular change in the behaviour is observed. For instance, a flattening out trend is observed (at about iteration 1800-2000) in the decrease of the average number of used words. Again such a situation can be attributed to the ongoing problem of events with low probability.

The fourth stage is a stabilisation phase, where all of the aforementioned measures, and as such the entire process, stabilises at a maximal coherence state. In particular, all agents share the same naming convention and can successfully communicate with each other. Moreover, once a formmeaning pairing is established and shared (within the population), i.e. all agents utilise the same naming convention, every future interaction results in no particular change in the lexicon, i.e. no other form-meaning pair can dominate the 
lexicon. For instance, the coherence rate reaches its maximum (1), average language words strength reaches its maximum (1), and the average number of used words stabilises at the value equal to the number of objects in the environment (10), i.e. establishing a one-to-one lexicon. It should be underlined that once the system enters such a state without any external perturbation, it cannot leave it, i.e. it is a stable state of the system.

In particular, we note that the underlying behaviour of the system is similar to both of the ICSL and INT strategies (see Fig. 3 and Fig. 5) and is perfectly in line with the results obtained by other researchers. For instance, the basic difference between the settings with global word creation (without unique words) and settings with local word creation (with unique words) is of the same nature, i.e. the initial rapid increase of coherence that swiftly decreases and the substantial difference in the maximal number of used words. Moreover, it also represents the four stage dynamics, where the initial associations are established at first. Next, the initial hooks are further developed and accompanied by novel words, and consequently new form-meaning paring possibilities. After reaching a full lexicon, i.e. when there is no need to introduce new words: all of the available objects are already named by all of the agents, the available space of possible associations is pruned, i.e. dominating associations (due to the particular realisation of the probabilistic nature of interactions) are enforced. Further, the pruning continues at a lowering rate with the ongoing interactions, as the less probable events are needed to complete the alignment. Finally, as in the aforementioned approaches, the process stabilises at a maximum coherence rate with one-to-one lexicons shared among the interacting agents.

Concluding, we can note some basic properties of the pair-wise interaction pattern (with ICSL strategy - see Fig. 3 and Tab. 1), i.e. the number of used words: $20-21$ after $1300-$ 1500 iterations without unique words and 36-37 after 8001000 iterations with unique words, total number of words 21-23 without unique words and 38-40 with unique words, average number of invented words $-10-12$ without unique words and 6-7 with unique words, and coherence rate of 0.8 after $1400-1600$ iterations with unique words and 17001900 iterations without unique words, of 0.9 after roughly 1700-1900 iterations with unique words and 2000-2200 iterations without unique words, and full coherence after around 2500-2700 iterations with unique words and 3000-3200 iterations without unique words. For the sake of completeness, we also note the basic properties of the pair-wise interaction pattern (with INT strategy - see Fig. 5), i.e. the number of used words: $20-21$ after 1200-1400 iterations without unique words and 36-37 after 900-1000 iterations with unique words, total number of words - 25-26 without unique words and 43-44 with unique words, average number of invented words - 12-13 without unique words and 6-7 with unique words, and coherence rate of 0.8 after $1500-1800$ it- erations, of 0.9 after roughly 1900-2100 iterations, and full coherence after around 3000-3200 iterations. These baseline values will be further used as reference points for the extended model settings.

\section{2. Multi-Participant Pattern - multiple speakers}

We begin by studying the case of multiple speakers (see Fig. 4). Just to clarify, the number of speakers indicates how many agents in a single interaction play the role of the speaker with a single fixed hearer. At first, introduction of multiple speakers results in significant gain in terms of performance (iteration-wise) that slowly diminishes as more agents are involved in the role of the speakers. Yet there is a drastic decrease in the number of iterations needed for the alignment processes to reach a particular level of coherence. For instance, in a settings where only two speakers are involved in interaction full coherence requires on average 1400 iterations, whereas with five speakers this requirement decreases to an average of 750 iterations (nearly half). We should note that the proper interpretation of the gain is not straightforward. In particular, as each iteration involves multiple acts of learning episodes it is somewhat unclear whether there is a particular decrease. On the other hand, the internal communications within a single interaction are bounded to a particular context and a single hearer as such they do not represent the full diversity of the original settings. Consequently, we tend to treat the observed behaviour as a particular gain in the alignment processes.

Using the Levenberg-Marquardt algorithm to solve the least squares curve fitting problem, we can try fitting a power function $a x^{b}+c$ to the obtained scaling property (the number of iterations needed to reach full coherence in the changing number of subiterations). We can note that for Int strategy the resultant is roughly $1550.77 x^{-1.196}+521.93$ and the fit is really good, i.e. the mean least square error is around 17.45 (69.80 in total). Moreover, the obtained fits for other strategies resemble a more significant error.

Further we can note that with the increasing number of subiterations there is a resultant increase in the number of words (see Fig. 6). In particular, the more speakers there are, in a single context setting, the more words need to be stored in the system, and also more words are used. This increase is due to the underlying increase in the number of iterations a particular agent plays the role of a speaker in its initial phase, i.e. where the lexicon is or is nearly empty. Consequently, the agent is required to invent new names for the yet unnamed objects. Moreover, the agents rarely play the role of a hearer, as compared to the number of times a word is uttered. These all aspects of the situation lead to the observed increase in the needed words with the increasing number of speakers per interaction.

\section{3. Multi-Participant Pattern - multiple hearers}

Next, we turn our attention to the settings where multiple hearers engage in a single interaction. In particular, we 

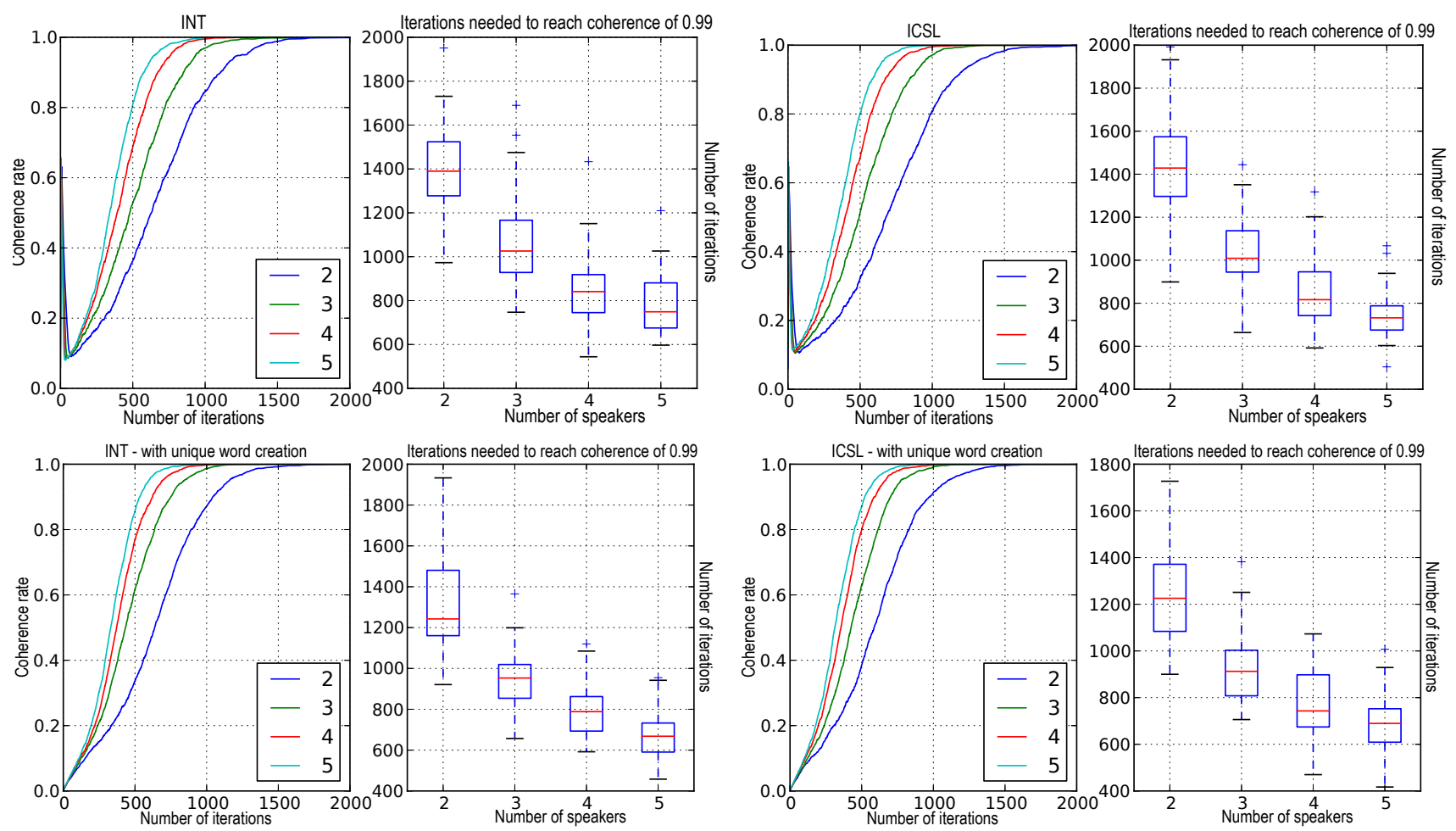

Fig. 4. Multiple Speakers (Coherence Rate) - Int (left column), ICSL (right column) without unique words (bottom row) and with unique words (top row)

assume that a single speaker broadcasts its word to a given set of hearers that all share the same current state of the environment: the context. Such a case resembles a relatively common situation in embodied multi-agent systems, where a group of individual system units meet in a particular spot and engages in communication. Still, from the point of view of the language game model these settings again fall outside of the original formulation and represent a particular formulation of the problem that was left out in the current research.

First we should note that due to the lack of a feedback loop between the speaker and the hearer (as there is no additional signalling pattern available to the agent) at a single episode only the hearer modifies its lexicon (despite a few situations where the speaker invents novel words). Consequently, increasing the number of hearers in a single episode increases the number of concurrent changes in the lexicons within the population. As such we can predict that the more hearers are engaged in a single interplay, the better the performance of the alignment processes is. Precisely, the obtained results (see Fig. 8) perfectly follow the aforementioned behaviour. In particular, the increasing number of hearers involved in a single interaction results in an increasing gain in terms of the number of iterations needed to reach a particular coherence rate. For instance, settings with two

Tab. 1. Pair-wise interaction pattern

\begin{tabular}{l|c|c||c|c}
\hline & \multicolumn{2}{|c||}{ ICSL } & \multicolumn{2}{c}{ INT } \\
\hline & unique & non-unique & unique & non-unique \\
\hline $\max \left(\mu_{U W}\right)$ & $20-21$ & $36-37$ & $20-21$ & $36-37$ \\
iter. to max $\left(\mu_{U W}\right)$ & $1300-1500$ & $800-1000$ & $1200-1400$ & $900-1000$ \\
$\mu_{T W}$ & $21-23$ & $38-40$ & $25-26$ & $43-44$ \\
\hline$\mu_{L C}=0.8$ & $1400-1600$ & $1700-1900$ & $1500-1700$ & $1600-1800$ \\
$\mu_{L C}=0.9$ & $1700-1900$ & $2000-2200$ & $1900-2100$ & $2100-2200$ \\
$\mu_{L C}=1$ & $2500-2700$ & $3000-3200$ & $3000-3200$ & $3200-3400$ \\
\hline
\end{tabular}



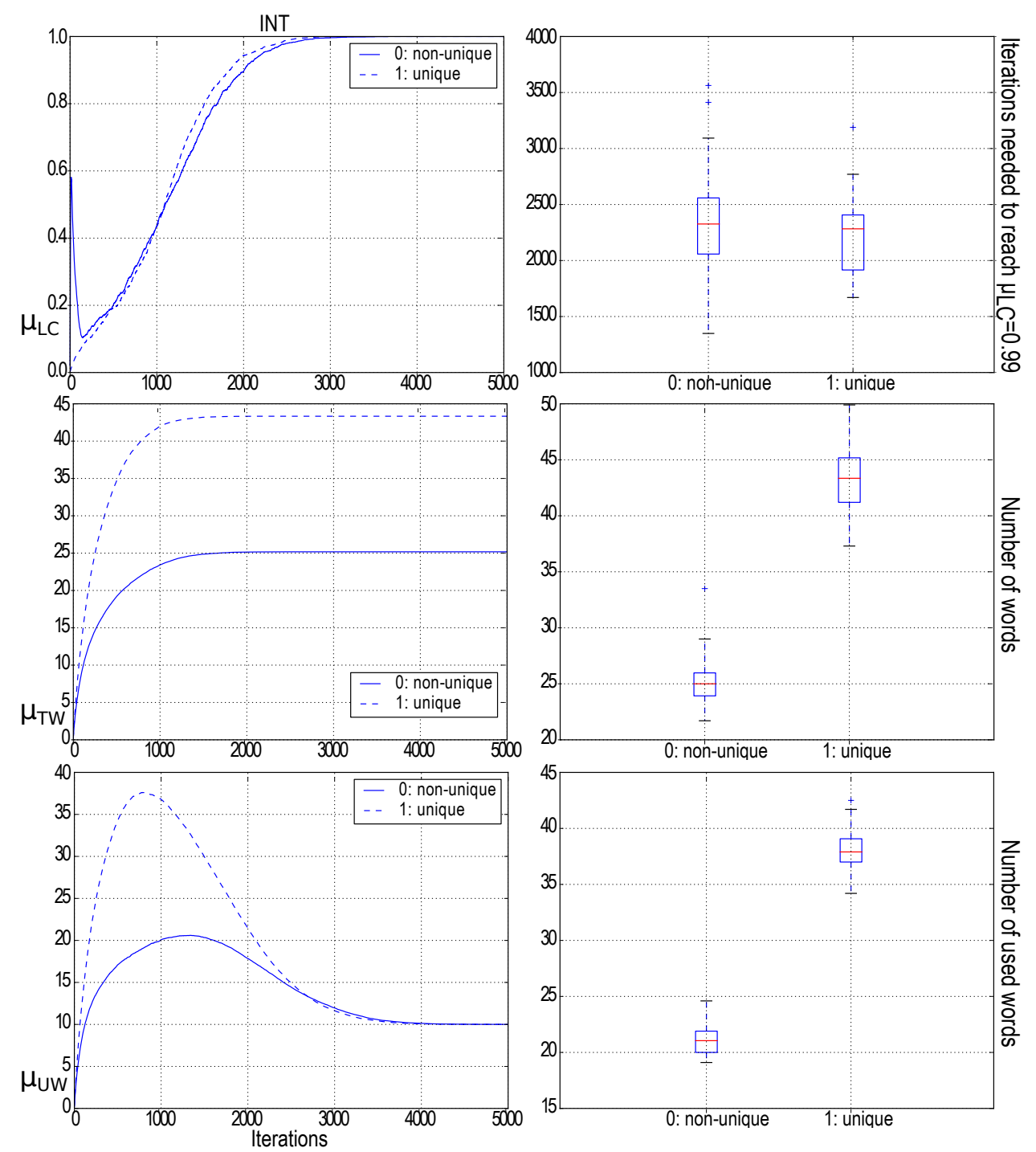

Fig. 5. Single-stage with single participants (INT) - coherence dynamics (top row), number of words (middle row) and number of used words (bottom row)

hearers require roughly $1000-1200$ iterations, whereas settings of five hearers require around 200-300 iterations (4-5 times less).

Moreover, the studied settings also affect the word wise performance. In particular, the increasing number of agents results in a significant decrease of the number of words used and needed by the population. This is a direct consequence of the fact that, opposite to the multiple speaker settings, it is relatively easy to be a hearing agent than a speaker. Further, as only the speaker can invent novel words it is more probable that a particular agent will engage in an interaction concerning a given object as a hearer than speaker. Consequently, as it already registered a word for that object there is no need to invent a new one. Certainly, after multiple interactions and due to the synonymy dampening procedures the agent might end up introducing a novel word. However, still this early explosion of words in the original settings is significantly bounded in the case of multiple hearers.

\section{4. Practical Aspects}

Concluding, we can note that from the alignment's process perspective it is preferred to have multiple hearers engaging in a single interaction rather than multiple speakers, i.e. in terms of iteration-wise performance (see Fig. 11). This follows the common-sense interpretation of such settings, as the ability of the system to perform local broadcast rather than one-to-one communication. Nevertheless, such a situation where multiple agents need to engage in the same episode is hard to be maintained in a real life application scenario. Nevertheless, we can also note that performance 

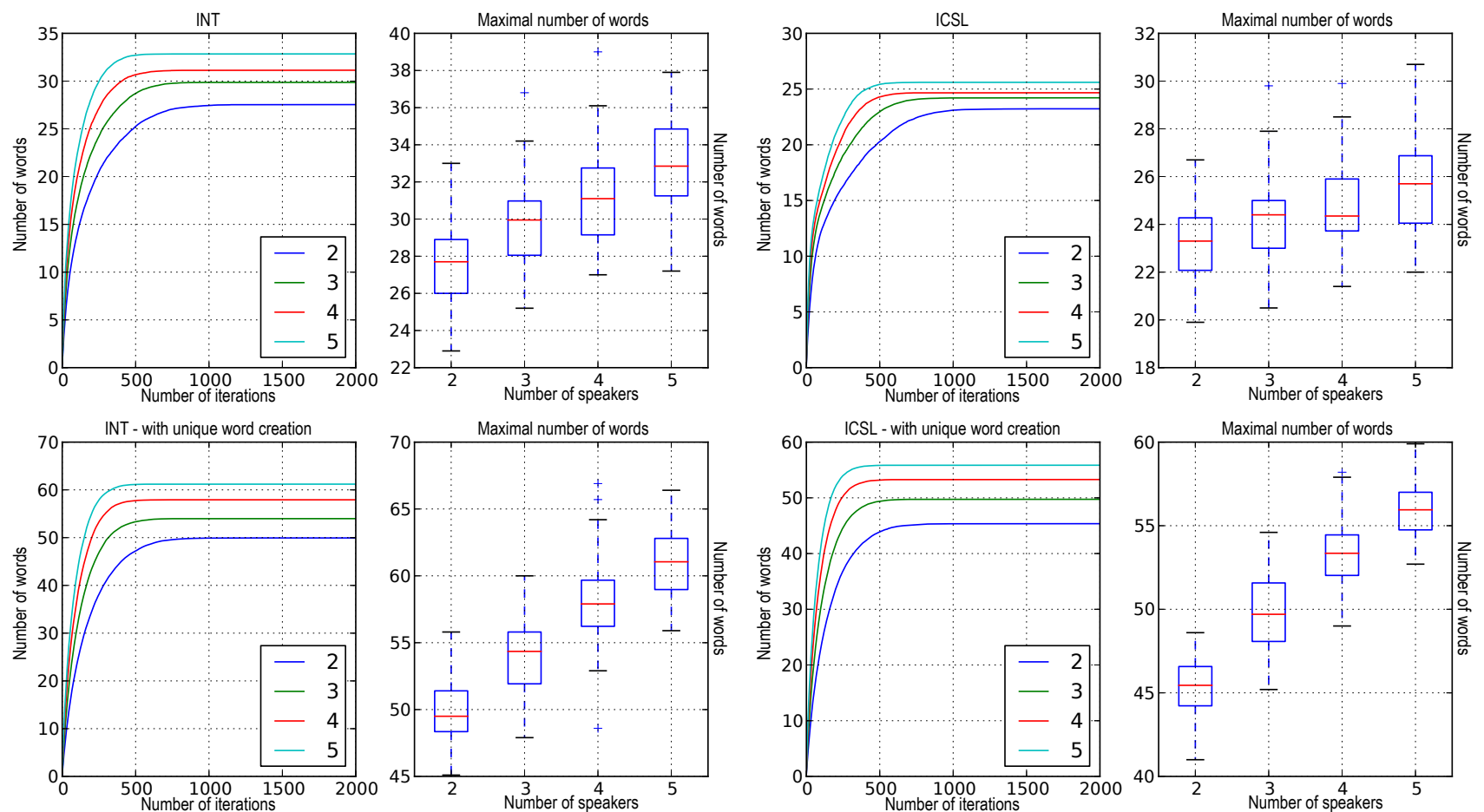

Fig. 6. Multiple Speakers (Number of words) - Int (left column), ICSL (right column) without unique words (top row) and with unique words (bottom row)
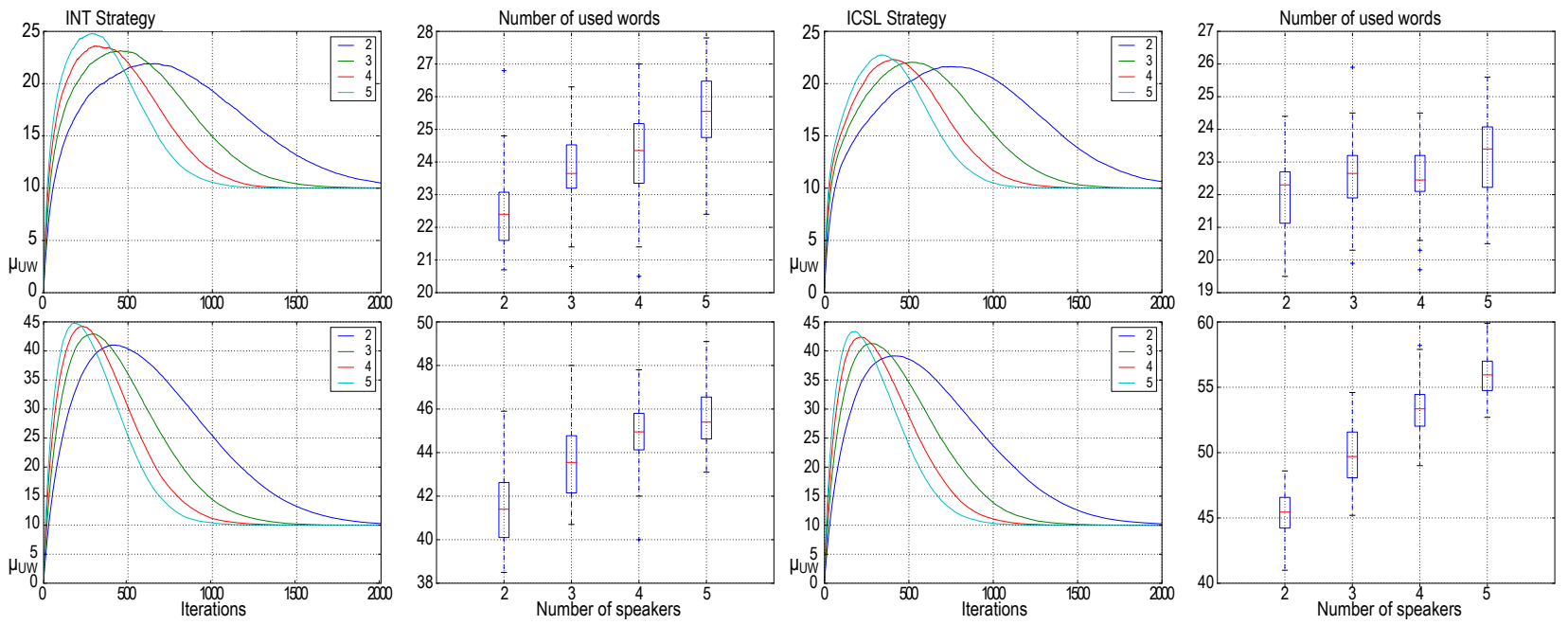

Fig. 7. Multiple Speakers (Number of used words) - Int (left column), ICSL (right column) without unique words (top row) and with unique words (bottom row)

wise it is better to involve multiple hearers in a single interaction rather than engage them in multiple dialogue-like interactions (see Fig. 11).

From a practical point of view, we can distinguish several important aspects of the language alignment processes in an extended model of communication. The pragmatic approach is directly related to a particular design task in which a de- signer (of an embodied multi-agent system) needs to address a few basic questions related to the alignment processes.

The designer needs to evaluate whether the settings of the embodied system allow the population of autonomous agents to develop a coherent naming convention and whether the development can be realised in a given time frame and with given resources. If these settings are in line with the re- 

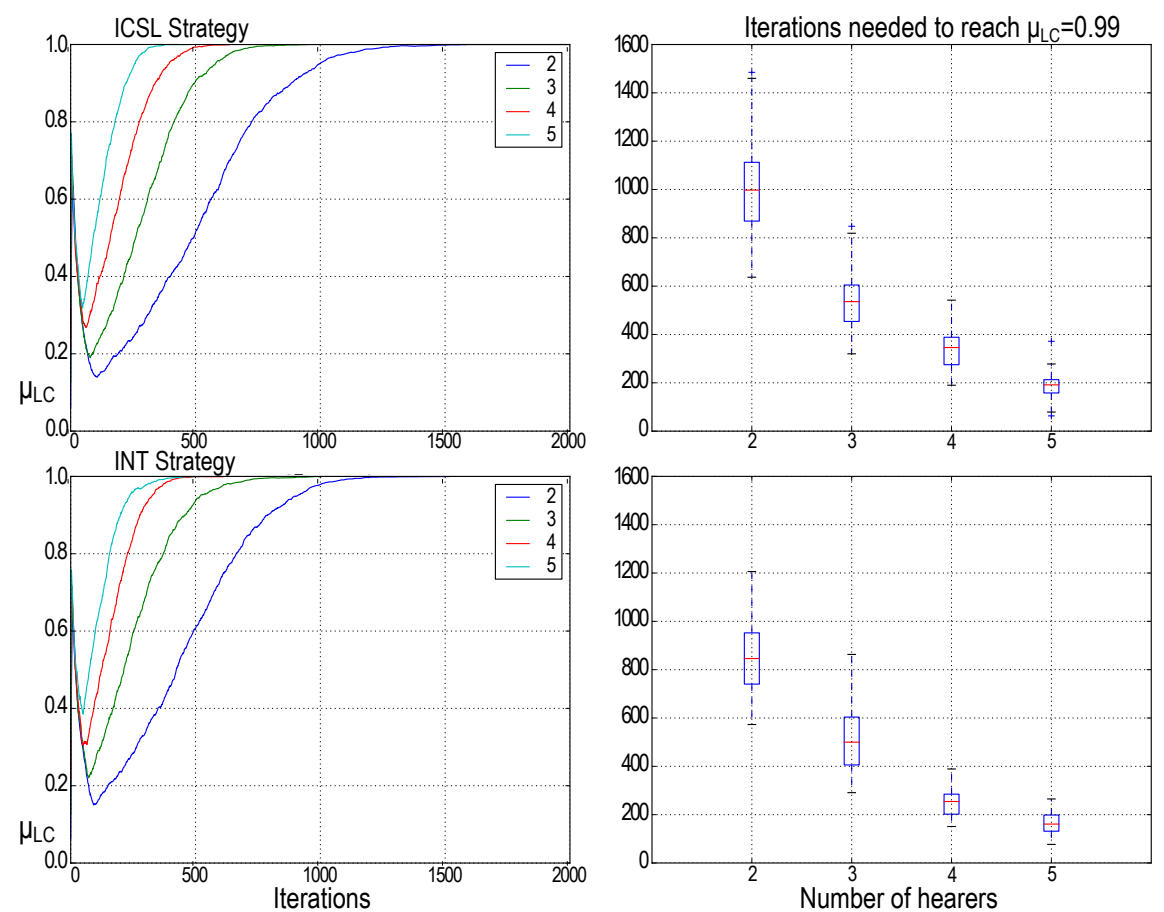

Fig. 8. Multiple Hearers (language coherence rate) - ICSL (top) and Int (bottom)
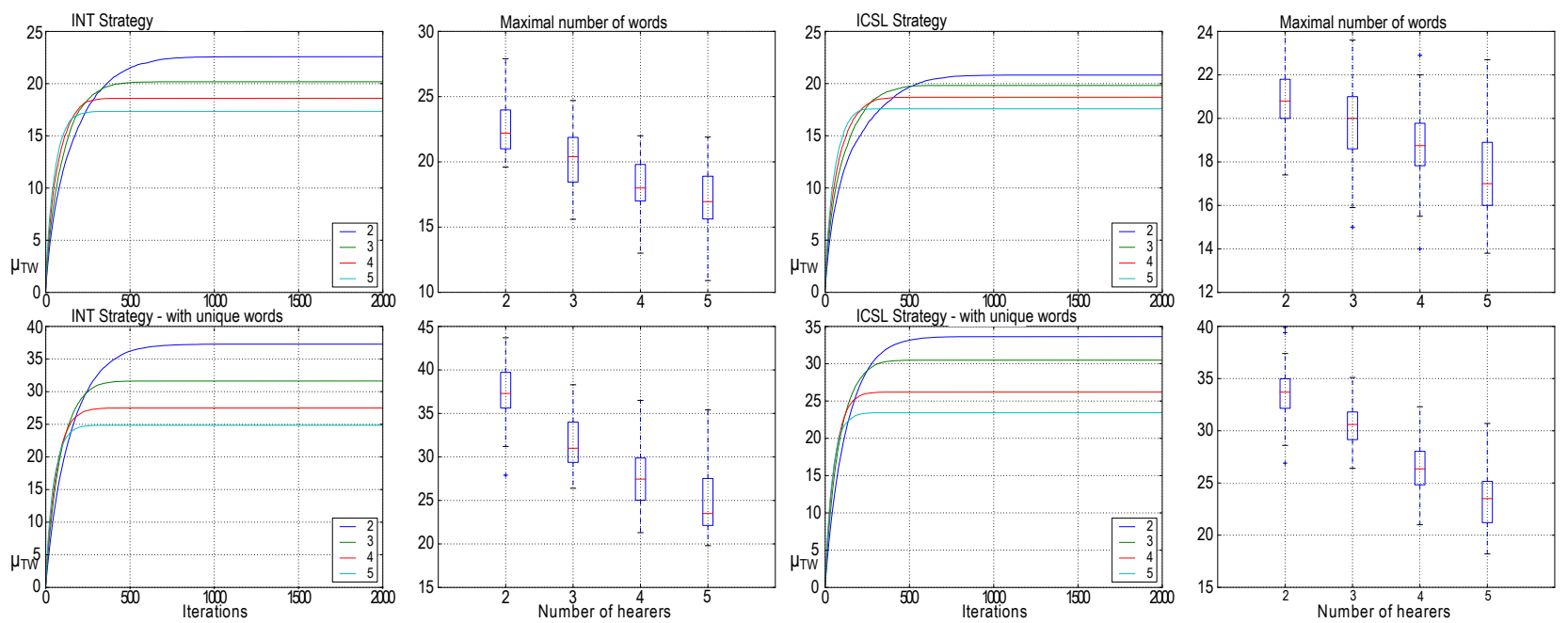

Fig. 9. Multiple Hearers (Number of words) - Int (left column), ICSL (right column) without unique words (top row) and with unique words (bottom row)

quirements defined by the LGM, then the situation is simple. Otherwise, for instance in a situation in which a strict singlestage and pair-wise interaction cannot be enforced, the designer is compelled to perform additional evaluation. From such a point of view, the presented research gives the designer valuable insights into the alignment processes in the extended settings, i.e. multi-participant interaction.
We can note that in terms of time-related aspect of the alignment processes allowing the system to engage multiple hearers and/or speakers in an interaction can significantly lower the number of interactions needed to reach a certain coherence (Sec. V. 2. and Sec. V. 3.), etc. However, in terms of resource consumption aspect of the alignment processes allowing the system to engage multiple hearers in an interaction can significantly lower these demands (Sec. V. 3.), etc. 

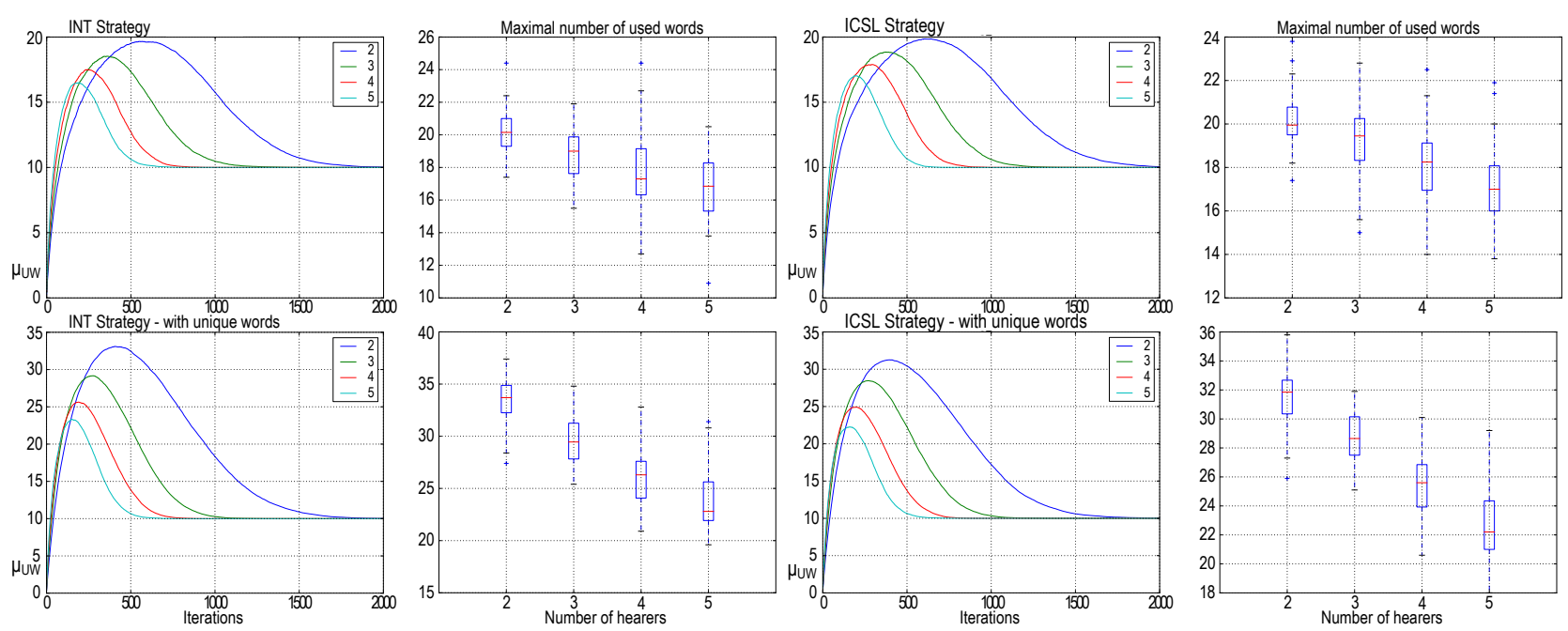

Fig. 10. Multiple Hearers (Number of used words) - Int (left column), ICSL (right column) without unique words (top row) and with unique words (bottom row)
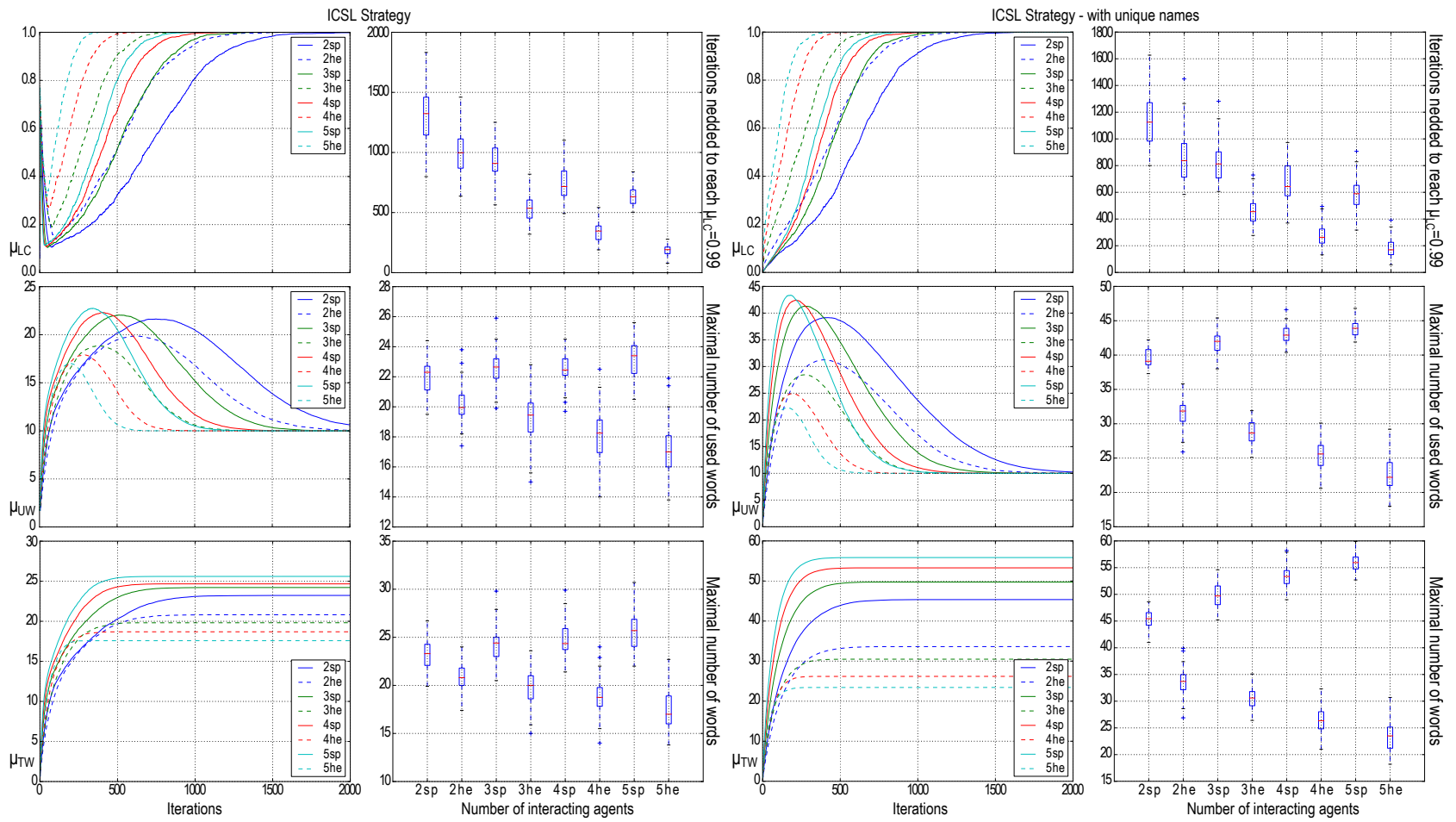

Fig. 11. Comparison (ICSL with 2-5 hearers or 2-5 speakers) - coherence rate (top row), number of words (middle row) and number of used words (bottom row) dynamics without unique word creation (left column) and with unique word (right column) creation pattern

\section{CONCLUSIONS}

In this paper we focused on the extended settings of the naming game without feedback, i.e. settings that are outside of the original formulation of the LGM.

We provided a concise meta-model of the multi-agent system capable of aligning a simple object-language. Most importantly, the introduced model covers the classical formulation of language game and allows for a set of certain extensions that fall outside of the original formulation of the LGM. As such, it provides a general structure of a system capable of involving in a naming game. 
The extension we focused on involves a multi-participant interaction pattern in a single episode of the naming game. We study the influence of different interaction patterns on the performance of the alignment processes. In particular, we managed to test the performance in a basic pair-wise interaction pattern against extended interaction patterns of multiple hearers and multiple speakers.

We showed that the extended settings of multiple hearers/speakers communication pattern still result in an ability of the multi-agent system to reach a coherent naming convention. Moreover, the dynamic behaviour of the alignment processes is of a similar nature. Nevertheless, the extended settings of multiple hearers/speakers communication pattern result in a significant gain in terms of performance (iterationwise performance). In particular the increasing number of speakers in a fixed context settings results in a decrease of the number of iterations needed to reach a particular level of coherence. Moreover, the increasing number of hearers in a fixed context settings results in an even greater decrease of the number of iterations needed to reach a particular level of coherence. However, such a gain in the multiple speakers case is possible at the cost of the increased number of words, thus requiring additional memory resources and greater computation power. In general, we can note that the more open a society of agents is, i.e. the agents are oriented towards hearing, the quicker the alignment processes are.

It should be mentioned that the presented simulationbased analysis is solely focused on the effects of different interaction models on the dynamic behaviour of the alignment processes. As such, the performed evaluation involved only an isolated study of different interaction patterns, whereas a more complex study could extend the presented approach to a case of non-idealised perception settings, non-uniform selection strategies and/or non-uniform environment characteristics.

\section{Acknowledgments}

This research was supported by Grant no. N N519 444939 funded by Polish Ministry of Science and Higher Education (2010-2013).

\section{References}

[1] A. Baronchelli, V. Loreto, L. DallAsta, and A. Barrat, Bootstrapping communication in language games: Strategy, topology and all that, [in:] Proceedings of EVOLANG 2006, World Scientific, 2006.

[2] P. Berger, and T. Luckmann, The Social Construction of Reality: A Treatise in the Sociology of Knowledge, Harmondsworth, Penguin Books, 1991.
[3] A. Cangelosi, The grounding and sharing of symbols, [in:] Cognition Distributed: How Cognitive Technology Extends Our Minds, 83, 2008.

[4] D. Cook and S. Das, How smart are our environments? An updated look at the state of the art, Pervasive and Mobile Computing 3(2), 53-73 (2007).

[5] B. DeVylder and K. Tuyls, Towards a common lexicon in the naming game: The dynamics of synonymy reduction, [in:] Workshop on Semiotic Dynamics of Language Games, 2005.

[6] J. DeBeule, B. DeVylder, and T. Belpaeme, A cross-situational learning algorithm for damping homonymy in the guessing game, [in:] Proceedings of ALIFE X, MIT Press, 2006.

[7] W.J. Freeman, The physiology of perception, Scientific American 264(2), 78-85 (1991).

[8] X. Hong, C. Nugent, M. Mulvenna, S. McClean, B. Scotney, and S. Devlin, Evidential fusion of sensor data for activity recognition in smart homes, Pervasive and Mobile Computing 5(3), 236-252 (2009).

[9] R. Katarzyniak, The language grounding problem and its relation to the internal structure of cognitive agents, Journal of Universal Computer Science 11(2), 357-374 (2005).

[10] J. Van Looveren, Multiple word naming games, [in:] Proceedings of BNCAI 1999, 1999

[11] W. Lorkiewicz, R. Kowalczyk, R. Katarzyniak, and Q.B. Vo, On topic selection strategies in multi-agent naming game, [in:] Proceedings of AAMAS 2011, International Foundation for Autonomous Agents and Multiagent Systems, 2011

[12] W. Lorkiewicz, R. Katarzyniak, and R. Kowalczyk, Individual semiosis in multi-agent systems, Transactions on Computational Collective Intelligence VII, 164-197 (2012).

[13] D. Lipowska. Naming game and computational modelling of language evolution Computational Methods in Science and Technology 17(1-2), 41-51 (2011).

[14] D. Lipowska, and A. Lipowski, Naming game on adaptive weighted networks, Artificial Life 18, 311-323 (2012).

[15] Q. Lu, G. Korniss, and B.K. Szymanski, Naming Games in Two-Dimensional and Small-World-Connected Random Geometric Networks, Phys. Rev. E 77(1), 016111, 1-10 (2008).

[16] M. Mirolli and S. Nolfi, Evolving communication in embodied agents: Theory, Methods, and Evaluation, Evolution of Communication and Language in Embodied Agents, 105-121, 2010.

[17] S. Nolfi, Emergence of communication in embodied agents: Co-adapting communicative and non-communicative behaviours, Connection Science 17(3), 231-248 (2005).

[18] I. Rekleitis, Distributed coverage with multi-robot system, [in:] Proceedings of ICRA'06, IEEE, 2006.

[19] L. Steels, Language Games for Autonomous Robots, IEEE Intelligent Systems 16, 16-22 (2001).

[20] L. Steels, Modeling The Formation of Language in Embodied Agents: Methods and Open Challenges, Evolution of Communication and Language in Embodied Agents, 223-233, 2010.

[21] P. Vogt and H. Coumans, Investigating social interaction strategies for bootstrapping lexicon development, [in:] Journal of Artificial Societies and Social Simulation 6(1) (2003).

[22] P. Vogt and B. De Boer, Language Evolution: Computer Models for Empirical Data, Adaptive Behavior 18(1), 5-11 (2010).

[23] K. Wagner, J.A. Reggia, J. Uriagereka, and G.S. Wilkinson, Progress in the Simulation of Emergent Communication and Language, [in:] Adaptive Behavior 11(1), 37-69 (2003). 


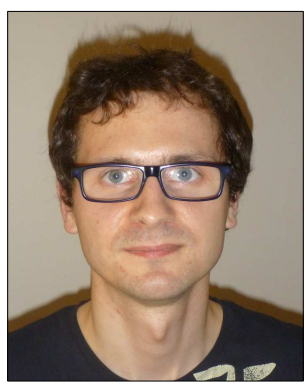

Wojciech Lorkiewicz Personal Data: born 02 March 1983, Wroclaw, Poland; Current Position: Research Assistant, Wroclaw University of Technology (WrUT), Institute of Informatics, Wroclaw, Poland; Member of Semantic Systems Engineering Group, WrUT; Academic Education: 2007: MSc in Computer Science from WrUT, 2013: PhD in Computer Science from WrUT, and Swinburne University of Technology, FICT, Melbourne, Australia; Professional Activities: Organising Committee member of several international conferences (IEA-AIE'08, ICCCI'09, ACIIDS'09, ICCCI'11, ACIIDS'11, ICCCI'12), Member of KES Focus Group on Agent and Multi-agent Systems; Author and co-author of about 20 research journal and conference papers; Current Research Interests: artificial intelligence - agent and multi-agent systems modelling; artificial cognition - semantic communication, language processing and evolution; knowledge management systems computational methods in knowledge processing and information retrieval.

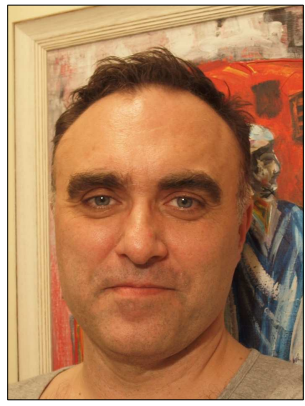

Radosław Katarzyniak Personal Data: born 01 June 1966, Kalisz, Poland; Current Position: Professor of Computer Science, Wroclaw University of Technology (WrUT), Institute of Informatics, Wroclaw, Poland; Head of Semantic Systems Engineering Group, WrUT; Academic Education: 1991: MSc in Computer Science from WrUT, Thesis: "Heuristics for Consensus Partition Problem."; 1999: PhD in Computer Science from WrUT, Thesis: "Multi-agent management in distributed processing systems."; 2009: D.Sc. (Habilitation) in Computer Science from AGH University of Science and Technology, Poland, Thesis: "Grounding of a Modal Language of Communication in Multiagent Systems."; Professional Activities: organizing chair of multiple conferences (IEA-AIE'08; International Conference on Collective Computational Intelligence ICCCI'09, ICCCI'10, ICCCI'11; Asian Conference on Intelligent Information and Database Systems - ACIIDS'09, ACCIDS'10); member of: SMCS Technical Committee on Computational Collective Intelligence and the Technical Committee of KES Focus Group on Agent and Multi-agent Systems; editor of a monograph "Ontologies and Soft Methods in Knowledge Management" (Int. Series on Natural and Artificial Intelligence, AKI, Adelaide), co-editor of five monographs on application of computational intelligence methods (Studies in Computational Intelligence, Springer); guest editor of special issues in international journals on multiagent systems and computational intelligence applications; author of about 120 research journal and conference papers; Current Research Interests: artificial intelligence - agent and multiagent systems modelling, semantic systems engineering; natural and artificial cognition - cognitive semantics, language processing, language evolution; knowledge management - computational methods in knowledge processing, information retrieval, and knowledge extraction. 